\title{
Tractions and Stress Fibers Control Cell Shape and Rearrangements in Collective Cell Migration
}

\author{
Aashrith Saraswathibhatla and Jacob Notbohm๑* \\ Department of Engineering Physics, University of Wisconsin-Madison, 1500 Engineering Drive, Madison, \\ Wisconsin 53706, USA
}

(Received 31 May 2019; revised manuscript received 12 November 2019; published 23 January 2020)

Key to collective cell migration is the ability of cells to rearrange their position with respect to their neighbors. Recent theory and experiments demonstrate that cellular rearrangements are facilitated by cell shape, with cells having more elongated shapes and greater perimeters more easily sliding past their neighbors within the cell layer. Though it is thought that cell perimeter is controlled primarily by cortical tension and adhesion at each cell's periphery, experimental testing of this hypothesis has produced conflicting results. Here we study collective migration in an epithelial monolayer by measuring forces, cell perimeters, and motion, and find all three to decrease with either increased cell density or inhibition of cell contraction. In contrast to previous understanding, the data suggest that cell shape and rearrangements are controlled not by cortical tension or adhesion at the cell periphery but rather by the stress fibers that produce tractions at the cell-substrate interface. This finding is confirmed by an experiment showing that increasing tractions reverses the effect of density on cell shape and rearrangements. Our study therefore reduces the focus on the cell periphery by establishing cell-substrate traction as a major physical factor controlling shape and motion in collective cell migration.

DOI: 10.1103/PhysRevX.10.011016

Subject Areas: Biological Physics, Mechanics, Soft Matter

\section{INTRODUCTION}

In numerous cases in human health and disease, epithelial cells transition from a static, motionless state to an active, migratory state. The active cell migration may generate new tissue, as in embryonic development, or further the spread of disease, as in cancer progression [1-3]. In either case, a critical step is the transition from motionless to migratory. Experiments have observed this transition [3-8] and demonstrated that it is akin to the jamming transition that occurs in particulate matter $[9,10]$. By analogy with the effect of density on jamming of rigid particles $[9,10]$, one might expect the transition from migratory to motionless in a cell collective to result from an increase in cell number density, and indeed this prediction has been observed in experiments [4,5,7, 11-14]. How the cell density affects the collective cell migration remains unclear, however, because the analogy between granular materials and cells is imperfect.

\footnotetext{
* Corresponding author. jknotbohm@wisc.edu

Published by the American Physical Society under the terms of the Creative Commons Attribution 4.0 International license. Further distribution of this work must maintain attribution to the author(s) and the published article's title, journal citation, and DOI.
}

In granular materials, the density-induced jamming transition is related to packing of particles and the free space available for motion, but cells within a monolayer cover all free space [15]. The mechanism causing jamming must therefore differ for cell monolayers and granular materials, though the differences remain unclear.

It may be that the effects of density on motion are indirect, which would mean that the motion is controlled by some other underlying physical factor. In support of this, experiments have observed a greater phosphorylated myosin light chain in epithelial monolayers of low density compared to high density [14], which is consistent with experiments that observed a proportional relationship between cell size and contractile stress [16,17]. This implies that density affects the active forces produced by each cell. Additionally, an increase in cell density due to cell division in epithelial monolayers coincides with maturation of cadherin-based cell-cell adhesions, which was proposed to reduce migration by increasing an effective friction between neighboring cells [7]. Thus, density may also affect adhesion between cells. However, there remains no clear link between density, adhesion, force, and motion.

A useful starting point to relate these various factors is a set of theoretical models relating the energy of each cell $E$ to the cell's area $A$ and perimeter $P$ according to the equation $E=K_{A}\left(A-A_{0}\right)^{2}+\xi P^{2}+\Gamma P$, where $K_{A}, A_{0}$, $\xi$, 
and $\Gamma$ are constants $[15,18-26]$. The variable $\Gamma$ is a line tension that acts much like surface tension in a fluid. It is thought to be controlled by the mechanical components at each cell-cell interface. More specifically, tension within the cell cortex increases the line tension, whereas cell-cell adhesion molecules decrease it $[6,15,18-27]$. The theory predicts that a parameter defining the average cell shape $q=P / \sqrt{ } A$ is a metric to determine whether the cell layer is solidlike, with no rearrangements between neighboring cells, or fluidlike, with cells easily rearranging and sliding past their neighbors $[6,21,28]$. As $q$ is linearly proportional to the cell perimeter, we refer to it hereafter as the cell perimeter.

Though experiments have confirmed the relationship between perimeter and migration [6], the interpretation of the theory is still unclear. First, as the cell perimeter is dimensionless, it is independent of cell size, and thus, it is unclear whether it is affected by density. Second, some implementations of the theory assume the line tension $\Gamma$ to be negative $[6,15,20,21,23-26]$, implying that the effect of cell-cell adhesions on the line tension is greater in magnitude than the effect of cortical tension. Experiments have not yet verified this assumption. Third, some experiments relating perimeter to motion in monolayers of cells also measured cell-substrate tractions and observed greater traction applied by fast-moving cells with larger perimeter $[6,28]$. The theory predicts that increased actomyosin contraction would increase cortical tension, thereby reducing the average cell perimeter and tending to diminish collective rearrangements. A possible resolution for this inconsistency is that traction itself may increase the cell perimeter and facilitate rearrangements. This has been suggested by a theoretical model that introduced a "selfpropulsion force," which is equivalent to traction [21], but this theoretical prediction has not been tested.

Here we compare the effects of line tension at the cell periphery and traction at the cell-substrate interface on the perimeter and rearrangements in monolayers of epithelial cells. In response to increased number density or decreased actomyosin contractility, cell traction, perimeter, and rearrangements decrease. We further show that by activating actomyosin contraction, the established relationship between density and rearrangement can be reversed. The commonality underlying all experiments is the relationship among tractions, cell perimeter, and rearrangements, in agreement with a theoretical prediction of the effect of traction on the perimeter and rearrangements [21]. Our results therefore point to traction as a major factor controlling both shape and motion in collective cell migration.

\section{RESULTS}

We begin with the well-established observation that cell density affects collective cell migration, with higher density tending to arrest rearrangements within a cell monolayer [4,5,7,11-14]. We seed Madin-Darby canine kidney (MDCK) type II cells into 1-mm micropatterned islands on polyacrylamide substrates with Young's modulus of $6 \mathrm{kPa}$ coated with collagen I. The cells are seeded to attain densities of approximately 1200 and 4200 cells $/ \mathrm{mm}^{2}$ by adjusting the seeding density but keeping the time of culture constant [Figs. 1(a) and 1(c)]. The cells are imaged over time, and cell trajectories show a difference in motion, with cells in high-density islands remaining stuck in place but cells in low-density islands moving more freely [Figs. 1(b), 1(d), 7(a), and 7(b)]. Notably, in low-density islands, collective swirls can be observed [Fig. 7(a)], indicating the presence of collective packs. For one pack to slide past another, cells must rearrange their local position with their neighbors. At the spatial resolution of our experiments, cell rearrangements cannot be tracked directly, so we infer cell rearrangements from two metrics of collective migration. First, we compute the mean-square displacement [MSD; see Eq. (1a)] of all cell trajectories in each island over a time interval of $120 \mathrm{~min}$ [Fig. 1(e)]. The MSD grows quadratically in time if cells move in a straight line; it grows more slowly than this if cell trajectories bend or curve, as will occur if cell motion is impeded by neighboring cells. We therefore fit the MSD to a function of the form MSD $\sim \Delta t^{\alpha}$ with the exponent $\alpha$ quantifying the cell motion. Consistent with previous studies [4,5,7, 11-14], the MSD for all cells in high-density islands has a smaller value of $\alpha$ than cells in low-density islands [Fig. 1(f)], indicating more constrained migration. We also analyze the MSD of individual cell trajectories averaging only over time $\left[\mathrm{MSD}_{i}\right.$; Fig. 7(c)]. The corresponding exponent $\alpha_{i}$ is significantly larger in the low-density islands [Fig. 7(d)], which provides additional evidence that more rearrangements are present in low-density islands compared to high-density islands. However, two different mechanisms could produce a large exponent $\alpha$ : (1) cells could freely rearrange with their neighbors or (2) a collective group of cells could move in a straight line. To verify that the differences in $\alpha$ result from differences in rearrangements rather than straight-line motion of collective groups, we use a second metric, the cage-relative MSD [CRMSD; Eq. (1b)], which analyzes the difference between each cell's displacement and the displacement of its nearest $N$ neighbors, thereby quantifying the local rearrangement between that cell and its neighbors [29]. The CRMSD is fit to CRMSD $\sim \Delta t^{\alpha_{\mathrm{CR}}}$ with the exponent $\alpha_{\mathrm{CR}}$ quantifying the cage-relative cell motion. As previous modeling and experiments showed cells to move in packs of size approximately 20 cells $[4,29]$, we vary $N$ from 4 to 20 cells and observe no significant change in $\alpha_{\mathrm{CR}}$ [Fig. 7(e)]. We therefore choose a pack size of $N=20$ cells for the remainder of our analysis. Compared to $\alpha, \alpha_{\mathrm{CR}}$ is smaller, but the trends are nonetheless the same, namely, that $\alpha_{\mathrm{CR}}$ is smaller in islands of higher density [Figs. 1(g) and 7(f)]. As both metrics for rearrangements $\alpha$ and $\alpha_{\mathrm{CR}}$ are decreased by increasing density, the data suggest 

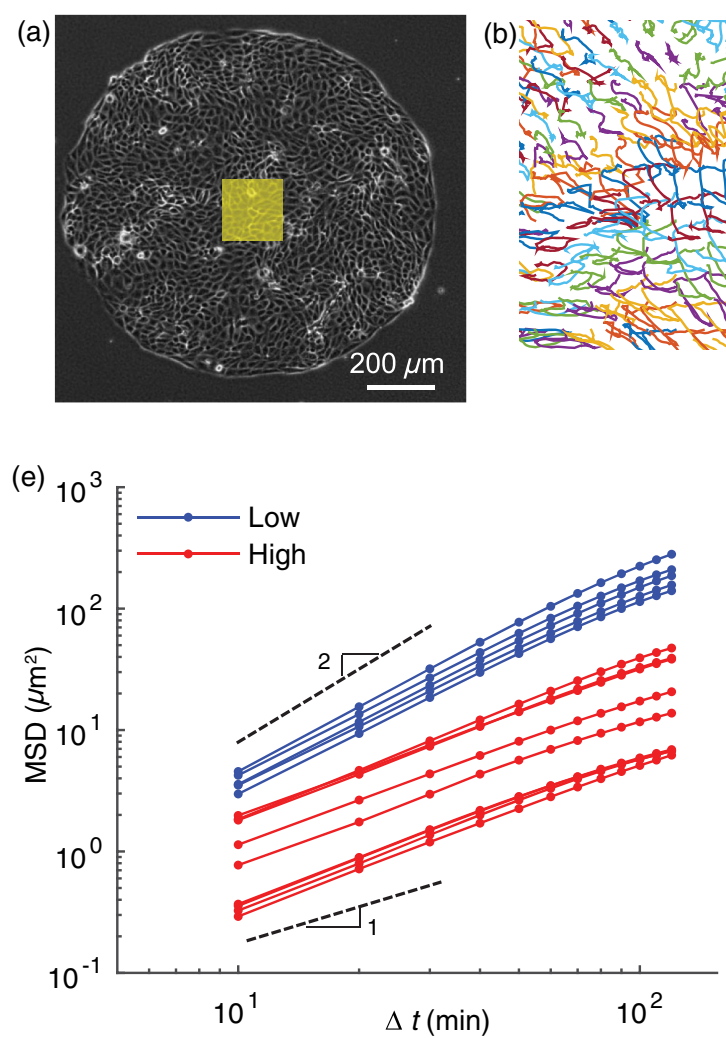

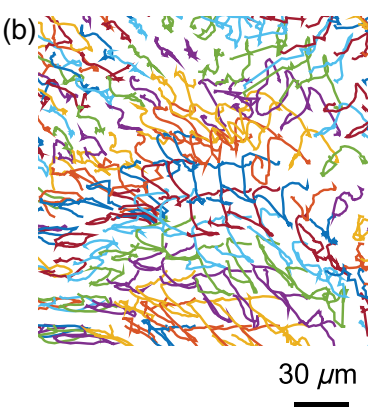

(c)

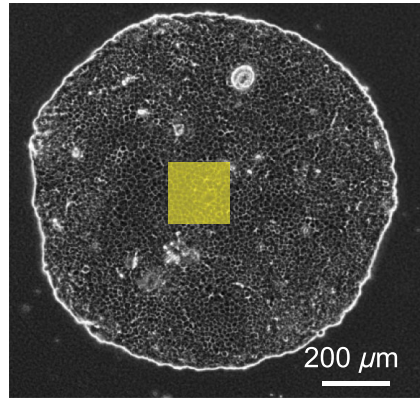

(f)

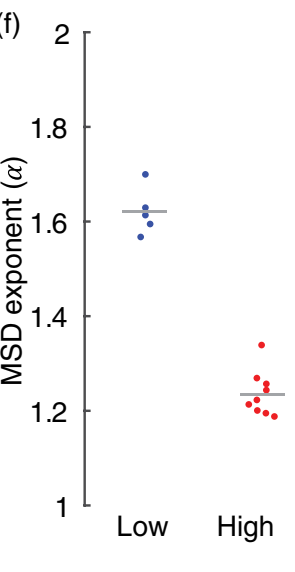

(g)

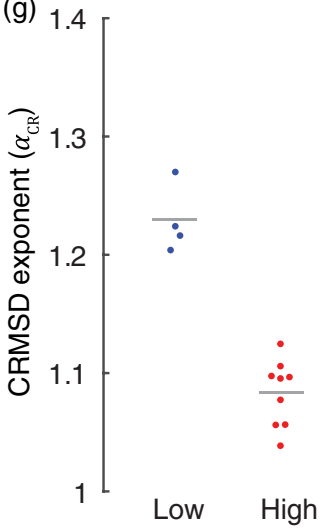

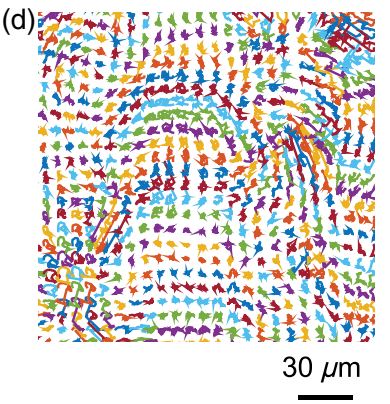

(h)

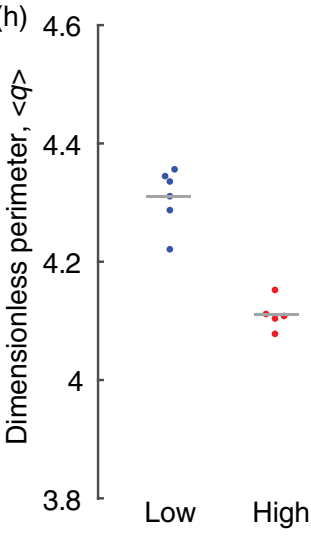

FIG. 1. Cell density decreases cell rearrangements and perimeter $q$. (a),(c) Phase contrast images of low-density (1200 cells $\left./ \mathrm{mm}^{2}\right)(\mathrm{a})$ and high-density (4200 cells $/ \mathrm{mm}^{2}$ ) (c) cell islands. (b),(d) Cell trajectories for the highlighted regions over $8 \mathrm{~h}$ in islands of low (b) and high density (d). Trajectories for the entire islands are shown in Fig. 7. (e) MSD for low- and high-density islands plotted on logarithmic axes. In this and all subsequent figures, each line of the MSD corresponds to a different cell island; dots represent the individual data points that make up the curve. (f) The MSD is fit to MSD $\sim \Delta t^{\alpha}$, with exponent $\alpha$ characterizing cell rearrangements. High-density islands have a smaller value of $\alpha$ than low-density islands $(p<0.001)$. In this and all subsequent figures, each dot of $\alpha$ corresponds to a different cell island. (g) Low-density islands have a larger exponent $\alpha_{\mathrm{CR}}$ than high-density islands ( $\left.p<0.001\right)$. (h) Average perimeter $\langle q\rangle$ is greater for cells at low density compared to high density $(p<0.001)$. In this and all subsequent figures, each dot of $\langle q\rangle$ corresponds to an average over 100-150 cells in a field of view, with at least two fields of view from at least three biologically different samples.

increasing density reduces rearrangements, as in previous studies $[4,5,7,11-14]$.

Consistent with previous experiments [6,28], the reduction in rearrangements also coincides with a decreased average perimeter $\langle q\rangle$ [see Sec. IV. for definition, Fig. 1(h)]. As in previous studies [8], the average cell aspect ratio shows the same trends as the perimeter, namely, that cells are more elongated in islands of low density than high density (Fig. 8). For the remainder of this manuscript, we focus on the perimeter $q$, as it is the variable considered by the theoretical models.

Because $q$ is dimensionless, it is unclear how it is affected by cell density, which has units of inverse area. For $q$ to change, the perimeter $P$ (having units of length) must scale nonproportionally with the square root of area $A^{1 / 2}$. We hypothesize that the nonproportional scaling results from a change in the distribution of forces controlling the cell perimeter and area. We begin by considering the forces at the cell periphery. According to current understanding $[6,15,18-27]$, a decrease in the perimeter $q$ is caused by an increase in the line tension $\Gamma$, which can result from an increase in cortical tension or a decrease in cell-cell adhesion.

To assess cortical tension, we fluorescently stain for phosphorylated myosin light chain [PMLC; Figs. 2(a) and 2(b)] indicative of actomyosin contractility, which generates cortical tension [27]. Using fluorescent images of actin [Figs. 2(c) and 2(d)] to detect cell peripheries [Fig. 2(e) and Sec. IV], we quantify fluorescent intensities of peripheral PMLC in cells at low and high density. Surprisingly, density has no apparent effect on peripheral PMLC intensity, suggesting no change in cortical tension [Figs. 2(a), 2(b), and 2(f)]. For a second measure of cortical tension, we perform laser ablation of cell-cell edges. The recoil of vertices previously connecting an ablated edge correlates positively to the tension in that edge before ablation and negatively to friction at that edge [30]. Consistent with this understanding, peripheral PMLC fluorescence and recoil velocity from laser ablation correlate in epithelial systems such as Caco-2 cells and 

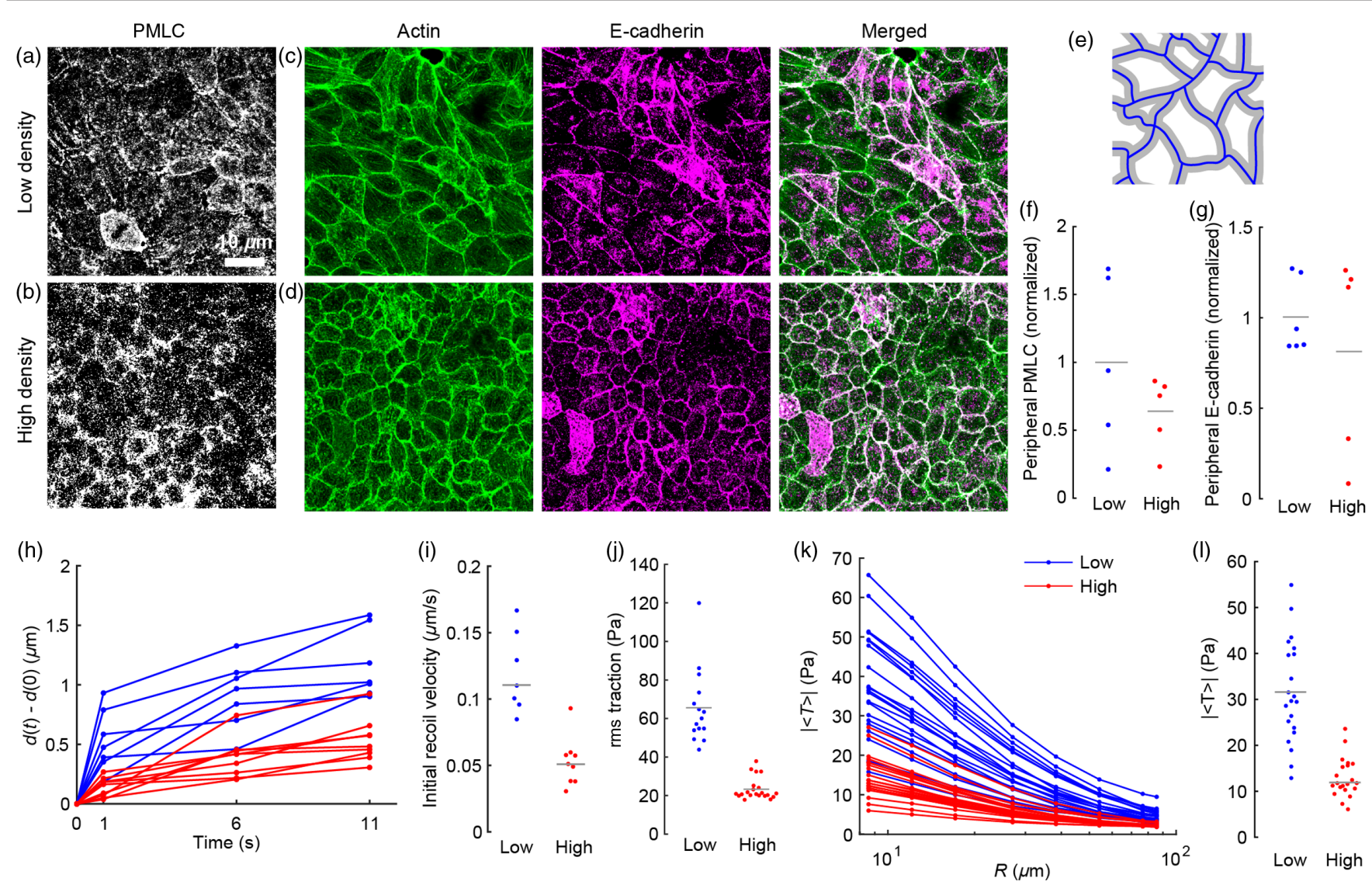

FIG. 2. Cell density has no effect on cortical tension or E-cadherin, but it decreases tractions. (a),(b) Confocal images of PMLC at low $\left(1200\right.$ cells $\left./ \mathrm{mm}^{2}\right)$ and high density $\left(4200\right.$ cells $\left./ \mathrm{mm}^{2}\right)$. (c),(d) Confocal images of actin and E-cadherin for cells at low (c) and high density (d). (e) Drawing showing analysis of fluorescent intensities at cell peripheries. Segmented cell boundaries (blue) are dilated by $1 \mu \mathrm{m}$ (gray); pixels within the dilated region are used for quantifying peripheral fluorescent intensities. (f) Fluorescent intensity of peripheral PMLC is unaffected by cell density $(p=0.4)$. For all data quantifying fluorescent images in this manuscript, each dot corresponds to an average over 100-150 cells in a field of view, with at least two fields of view from at least three biologically different samples. (g) Fluorescent intensity of peripheral E-cadherin is unaffected by cell density $(p=0.7)$. (h) Change in distance between the vertices of an ablated edge $d(t)-d(0)$ in cells at low and high densities. Each line corresponds to an ablated cell edge. (i) Cells at low density show higher initial recoil velocity compared to cells at high density $(p<0.01)$. For all data quantifying recoil velocity in this manuscript, each dot corresponds to a different ablated edge. (j) Average rms traction decreases with increase in cell density $(p<0.001)$. (k) Average traction imbalance computed over different circular regions of radius $R$. In this and all subsequent figures, quantifying traction or traction imbalance, each dot corresponds to a different cell island. (1) Average traction imbalance for $R=12 \mu \mathrm{m}$ is statistically larger in islands of low density than high density $(p<0.001)$.

Drosophila [31,32]. We ablate cell edges (see Video 1) and measure the distance $d(t)$ between the vertices that connect the ablated edge from 1 to $41 \mathrm{~s}$ after ablation. Data are reported by taking the change in distance $d(t)-d(0)$, where $d(0)$ is defined as the initial length of the cell edge before ablation. Most vertices retract linearly with time for $11 \mathrm{~s}$, after which they retract more slowly (Fig. 9). We therefore fit a line to the change in distance for $t=0$ to $11 \mathrm{~s}$ (gray region in Fig. 9) to compute the initial recoil velocity. Cells at low density show higher recoil velocity compared to cells at high density [Figs. 2(h) and 2(i)]. As there is no change in PMLC fluorescent intensity, this finding may suggest greater friction at cell-cell edges in islands of greater density as proposed previously [7]. It is also possible that laser ablation is more sensitive than PMLC fluorescence to line tension, implying that increasing density causes a decrease in cortical tension, in agreement with previous findings in epidermal progenitor cells [33].

We next consider cell-cell adhesion by fluorescent intensity of E-cadherin, which is associated with changes in cellcell contact areas and cell sorting [34,35]. Fluorescent imaging shows no difference in E-cadherin at low and high densities, implying no change in cell-cell adhesions [Figs. 2(c), 2(d), and 2(g)]. As the results suggest that increasing density has no effect on adhesion and causes either no change or a reduction in cortical tension, we conclude that increased density either has no effect on line tension $\Gamma$ or decreases it. Accordingly, the average perimeter $\langle q\rangle$ is expected to either remain the same or increase, but in contrast, increasing the density decreases $\langle q\rangle$ [Fig. 1(h)]. 


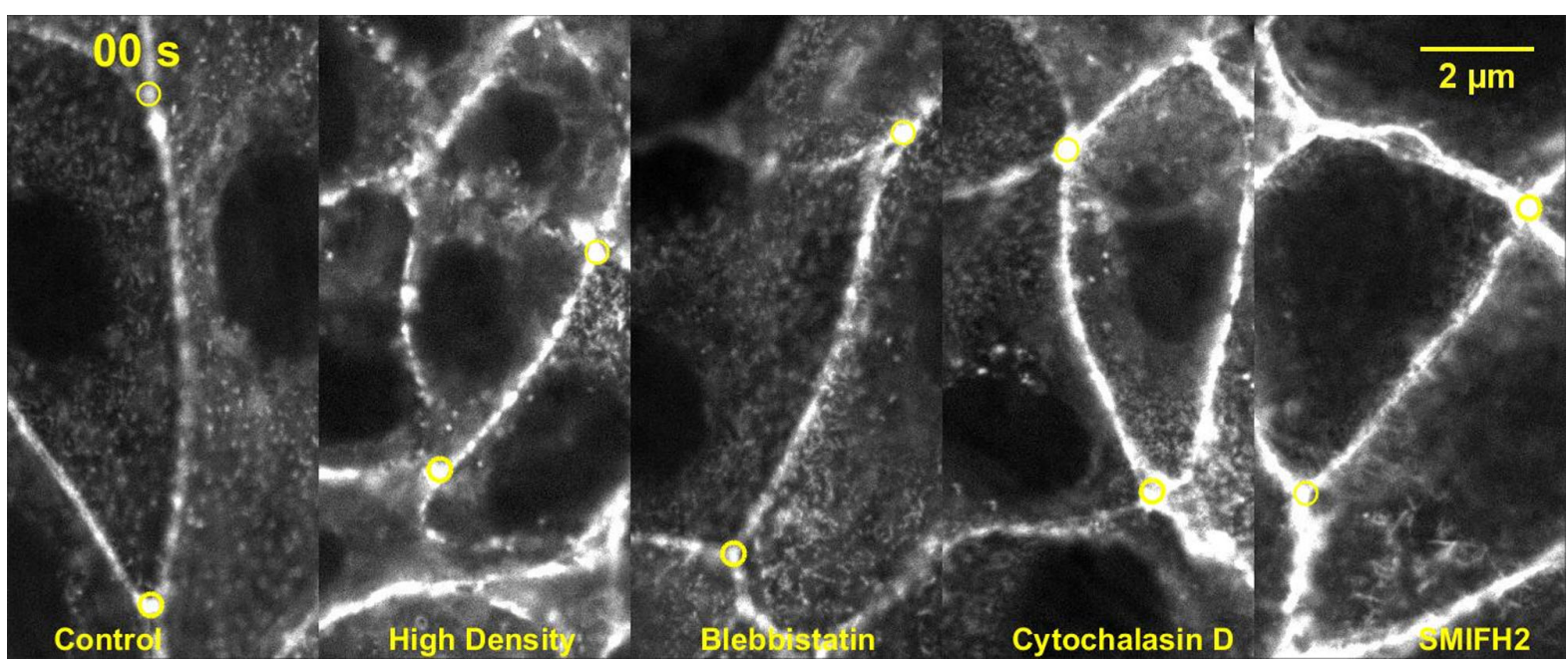

VIDEO 1. Laser ablation of cell edges. Time lapse imaging of actin at cell-cell edges immediately before $(t=0 \mathrm{~s})$ and after $(t>0 \mathrm{~s})$ laser ablation of control, high density, blebbistatin-treated, cytochalasin D-treated, and SMIFH2-treated cells. Circles indicate locations of vertices at $t=0 \mathrm{~s}$.

We then quantify traction at the cell-substrate interface, which has been proposed by a theoretical model to be another factor that increases the average perimeter $\langle q\rangle$ [21]. We measure tractions in the islands of different cell density using traction force microscopy [Figs. 10(a) and 10(b)] [36-38]. The root-mean-square (rms) tractions are larger in islands of low density [Fig. 2(j)], consistent with the inverse relationship between cell density and cell contraction observed in other studies [11,14,16,17]. As the rms gives an average over space, it does not necessarily quantify the traction that produces motion. It could be that the tractions produced by each cell balance such that the vector sum of traction applied by that cell to the substrate is zero. To account for this possibility, we define a traction imbalance (see Sec. IV), which quantifies the vector sum of traction for different circular regions of radius $R$. A nonzero traction imbalance implies stresses at the cell-cell interfaces [11,39] and is a driving force for motion. The average traction imbalance is larger in low-density islands than high-density islands [Fig. 2(k)], which is consistent with the trend in rms traction. A statistical test on the traction imbalance for $R=12 \mu \mathrm{m}$ shows a significant difference [Fig. 2(1)]. These findings hint that traction at the cell-substrate interface, rather than cortical tension or adhesion at the cell periphery, may be the physical factor controlling cell shape and motion.

To investigate further the effects of adhesion, cortical tension, and traction on shape and motion, we design experiments to modulate actomyosin contraction by treating with blebbistatin to disrupt myosin II and cytochalasin $\mathrm{D}$ to disrupt actin polymerization in islands of equal density (1200 cells $\left./ \mathrm{mm}^{2}\right)$. To investigate the effect of the inhibitors on cortical tension, we image the actin and PMLC at the cell peripheries [Figs. 3(a) and 3(c)]. Blebbistatin causes cell peripheries to become more curved [Figs. 3(a) and 3(b)], implying reduced cortical tension [28,31], whereas cytochalasin $\mathrm{D}$ decreases the amount of peripheral PMLC [Figs. 3(c) and 3(d)], also implying reduced cortical tension [31,32]. Additionally, recoil velocity decreases in response to either treatment [see Video 1 and Figs. 3(e)-3(h)], which, assuming no change in cell-cell friction, indicates reduced cortical tension. These observations are consistent with the fact that cortical tension results from actomyosin contractility [27]. To assess cell-cell adhesions, we fluorescently label E-cadherin. The treatments have no significant effect on Ecadherin intensities (Fig. 11), indicating no effect of the treatments on adhesion. Together, these findings suggest that actomyosin inhibition reduces the line tension $\Gamma$. According to current understanding [6,15,18-27], the reduced line tension should increase the average cell perimeters and the rearrangements. By contrast, the treatments reduce the cell perimeters [Figs. 3(i) and 3(j)]. Additionally, both inhibitors reduce the exponents $\alpha$ of MSD and $\alpha_{\mathrm{CR}}$ of CRMSD (Fig. 12), implying reduced cell rearrangements. These results contrast with current understanding that decreased line tension should increase cell perimeter and rearrangements, which suggests some other factor likely affects perimeter and rearrangements.

Tractions are also measured before and after inhibiting contractility with blebbistatin or cytochalasin D. In these experiments, the rms traction is measured before and after each treatment and normalized to the value before the treatment. The rms tractions are reduced by both treatments in a dose-dependent manner and remain nearly constant for 1 to $3 \mathrm{~h}$ after each treatment [Figs. 4(a), 4(b), 10(c), and 10(d)]. Averaging over this time window gives a single scalar measure of traction for each cell island. Statistical comparison shows that both inhibitors significantly decrease the rms traction [Figs. 4(c) and 4(d)]. Likewise, the traction imbalance is significantly reduced by the inhibitors [Figs. 4(e)-4(h)]. The data therefore suggest 


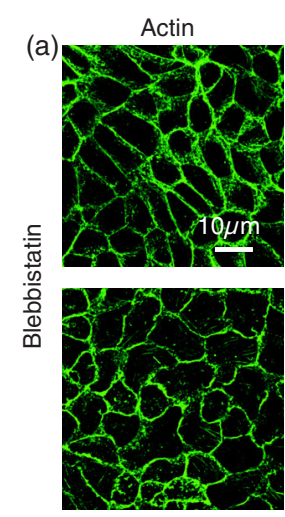

(b)
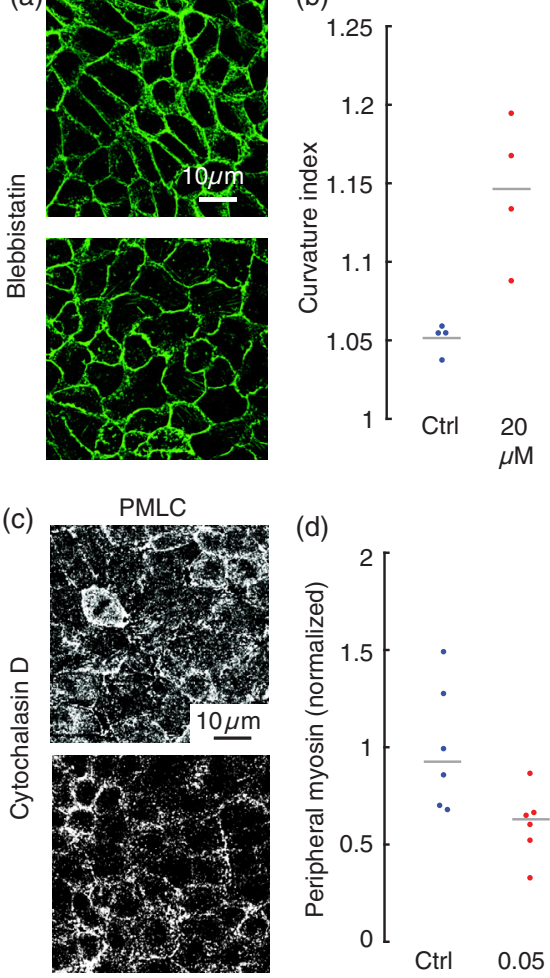

(d) (e)
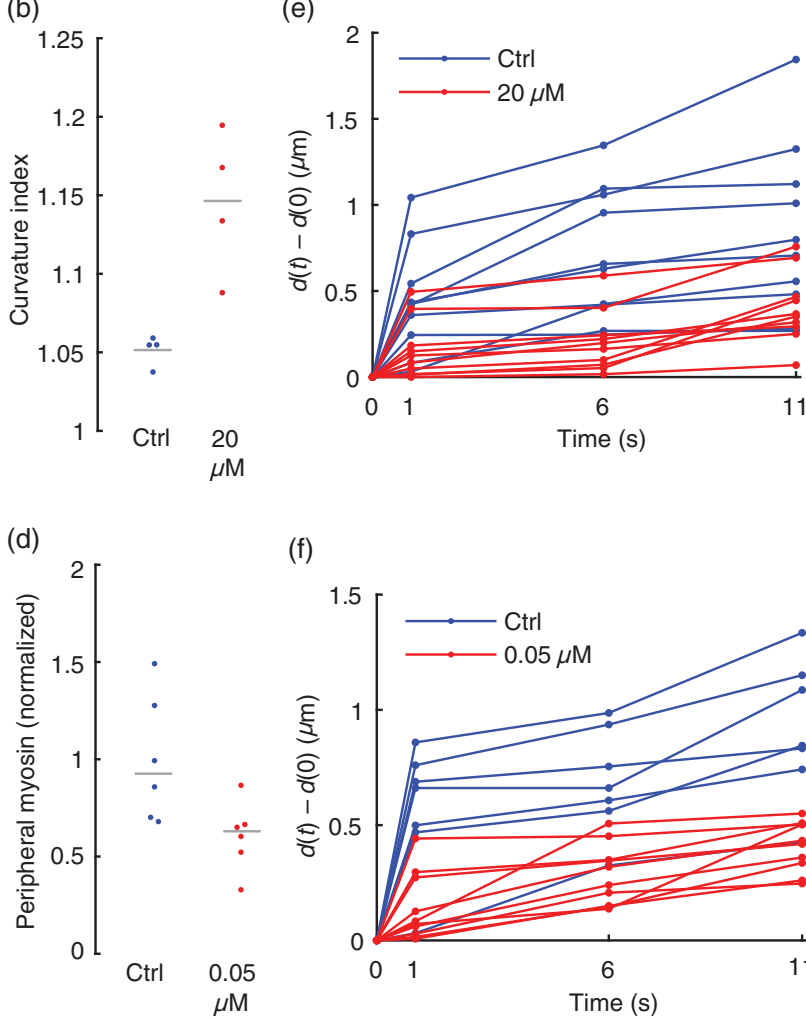

(f)

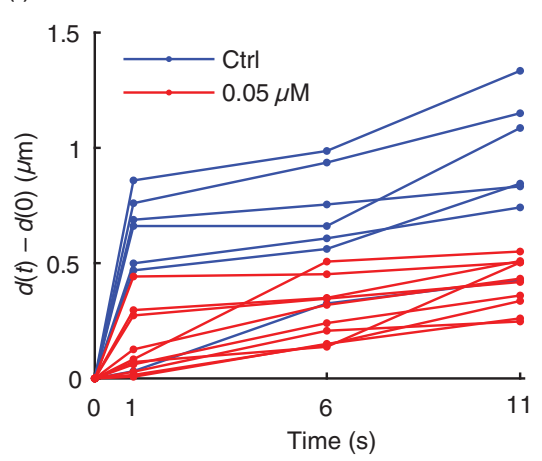

(g)

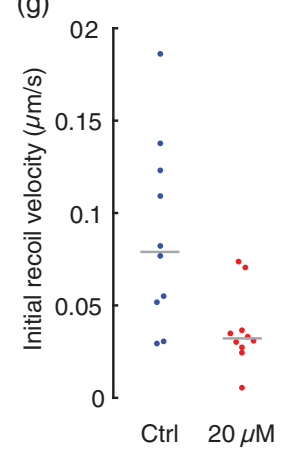

(i)

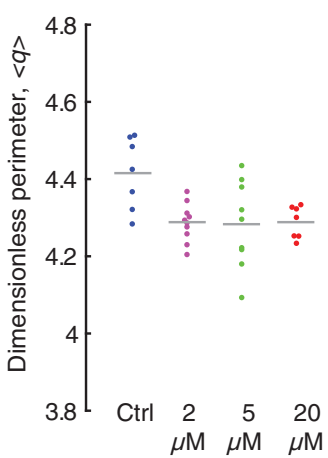

(h)

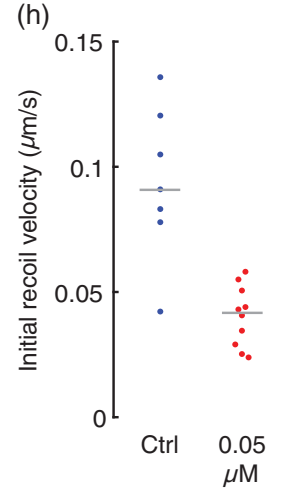

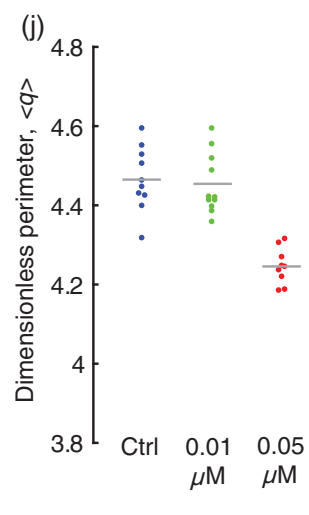

FIG. 3. Effect of blebbistatin and cytochalasin D on cortical tension and cell perimeter. (a) Cortical actin imaged for control cells (top) or cells treated with $20 \mu \mathrm{M}$ of blebbistatin (bottom). (b) Cell curvature index (defined as total edge length divided by end-to-end distance) increases after treating with $20 \mu \mathrm{M}$ of blebbistatin $(p<0.01)$. (c) PMLC imaged for control cells (top) or cells treated with $0.05 \mu \mathrm{M}$ of cytochalasin D (bottom). (d) Peripheral PMLC fluorescent intensity is decreased by cytochalasin D ( $p<0.05$ ). (e),(f) Change in distance between the vertices of an ablated edge $d(t)-d(0)$ for the control and cells treated with $20 \mu \mathrm{M}$ of blebbistatin (e) or $0.05 \mu \mathrm{M}$ of cytochalasin D (f). (g),(h) Initial recoil velocity decreases after treating with $20 \mu \mathrm{M}$ of blebbistatin $[p<0.01(\mathrm{~g})]$ or $0.05 \mu \mathrm{M}$ of cytochalasin D [ $p<0.001(\mathrm{~h})]$. (i), (j) Average perimeter $\langle q\rangle$ measured after treating with blebbistatin (i) or cytochalasin D (j) at different concentrations. Both $20 \mu \mathrm{M}$ of blebbistatin $(p<0.01)$ and $0.05 \mu \mathrm{M}$ of cytochalasin $\mathrm{D}(p<0.001)$ decrease $\langle q\rangle$. For all data in this figure, the cell density is approximately 1200 cells $/ \mathrm{mm}^{2}$.

that traction, rather than cortical tension or cell-cell adhesion, may affect cell perimeters and rearrangements. This is in contrast with the notion that perimeter and rearrangements result from cortical tension and cell-cell adhesion [6,15,18-27], but it is nevertheless consistent with a theoretical model that predicted traction, in addition to cortical tension and adhesion, can increase cell perimeter and rearrangements [21].

The notion that traction relates to the perimeter more so than cortical tension or adhesion is unexpected, because the forces of cell traction are not applied around the cell's periphery, as is cortical tension, but rather at the cell-substrate interface. The only logical explanation for tractions having a dominant effect on the perimeter is that the forces associated with traction must be far larger than forces of cortical tension and adhesion. This argument is supported by the fact that the amount of PMLC in the stress fibers is approximately 3.5 times that in the cortex (Fig. 13). To explore this idea further, we image stress fibers for cells in monolayers of high (approximately 4200 cells $/ \mathrm{mm}^{2}$ ) and low (approximately
1200 cells $/ \mathrm{mm}^{2}$ ) density. The images show a clear difference, with cells at high density having fewer and less aligned stress fibers [Figs. 5(a) and 5(b)], in agreement with the lower tractions produced [Fig. 2(i)]. To quantify stress fiber alignment, we compute an order parameter $S$ [Eq. (2)], which attains a value of 0 when stress fibers are randomly aligned and 1 when they are fully aligned. The average value of order parameter $S$ is 0.59 for cells at low density and 0.43 for cells at high density [Fig. 5(c)]. Similarly, both blebbistatin and cytochalasin D dramatically reduce the number of stress fibers [Figs. 11(a)-11(c)].

As a test of the relationship between stress fibers and perimeter, we use SMIFH2 to inhibit formins, which bundle actin filaments to form actin stress fibers [40]. Two concentrations (20 and $40 \mu \mathrm{M})$ of SMIFH2 decrease the traction by $60 \%$ and $65 \%$, respectively [Figs. 14(a) and 14(b)]. The treatment reduces stress fibers and their alignment [Figs. 5(d) and 5(e)] as quantified by the order parameter $S$ [Fig. 14(c)]. The effect of SMIFH2 on cell rearrangements is not quantified because the effects of 

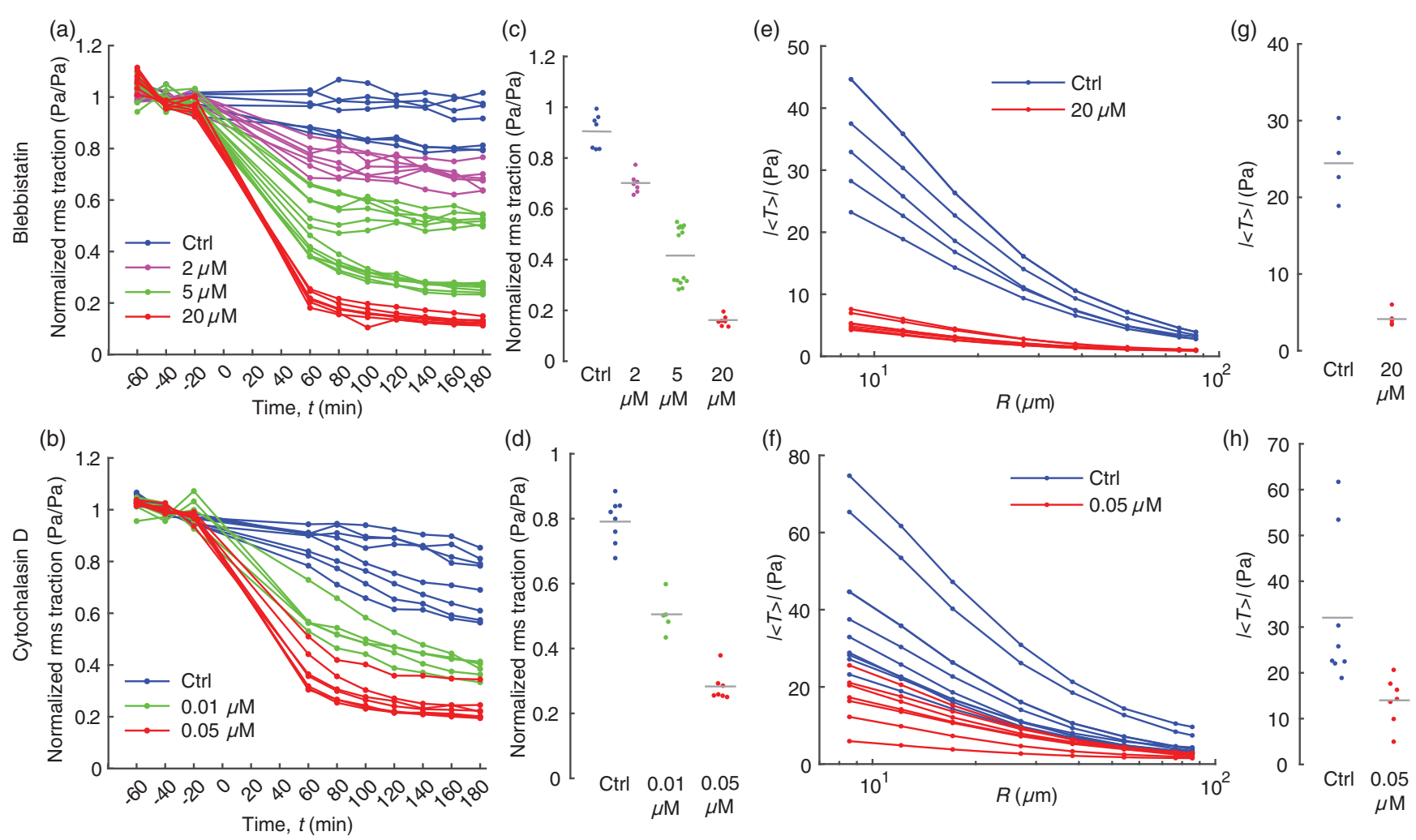

FIG. 4. Blebbistatin and cytochalasin D show dose-dependent reduction in tractions. (a),(b) Average rms traction for different cell islands measured over time before $(t<0)$ and after $(t>0)$ treating with blebbistatin (a) or cytochalasin D (b). For each island, the average rms traction is normalized by its average value before the treatment. (c),(d) Normalized rms tractions are averaged for $t=60-120 \mathrm{~min}$. All groups are statistically different from each other $(p<0.001)$. (e), (f) Average traction imbalance over different circular regions of radius $R$ for control islands and islands treated with blebbistatin (e) and cytochalasin D (f). (g),(h) The magnitude of average traction imbalance for $R=12 \mu \mathrm{m}$ is reduced by both blebbistatin (g) and cytochalasin D (h) $(p<0.001$ for both treatments compared to the control). For all data in this figure, the cell density is approximately $1200 \mathrm{cells} / \mathrm{mm}^{2}$.

SMIFH2 wear off after approximately $1 \mathrm{~h}$ of treatment [41]. Consistent with this timing, the tractions begin to increase $1 \mathrm{~h}$ after the treatment [Fig. 14(a)]. Nevertheless, the inhibitor decreases the dimensionless perimeter $\langle q\rangle$ [Fig. 5(f)], giving additional evidence of the connection between stress fibers and average perimeter $\langle q\rangle$. To rule out the possibility that SMIFH2 affects the cell perimeter by altering cortical tension, we perform laser ablation on cell edges and stain for PMLC. In agreement with previous findings [42], initial recoil velocity is lower in cells with SMIFH2 compared to the control, suggesting a reduction in cortical tension [Figs. 14(d) and 14(e)]. Surprisingly, PMLC fluorescent intensity at the cell peripheries increases [Fig. 14(f)], implying an increase in cortical tension. We suspect that the difference in observations between recoil velocity and PMLC is that formin inhibition can lead to disorganized F-actin at cell-cell junctions [42]. Therefore, even though there may be more PMLC at cell peripheries, the myosin may be disorganized and unrelated to cortical tension. Consistent with this explanation, laser ablation of SMIFH2-treated cell edges causes cortical F-actin to disperse rather than retract (see Video 1), suggesting disorganized cortical actomyosin. The SMIFH2 treatment also decreases E-cadherin expression at cell-cell junctions [Fig. 14(g)], consistent with the notion that forminnucleated actin at cell-cell junctions stabilizes cell-cell adhesions [42]. In summary, the effect of formin inhibition on line tension remains unclear, with PMLC fluorescence and E-cadherin imaging suggesting an increase in line tension and laser ablation suggesting a decrease. Nevertheless, the experiments with the formin inhibitor show a clear relationship between stress fibers [Figs. 5(d), 5(e), and 14(c)] and average perimeter $\langle q\rangle$ [Fig. 5(f)], giving additional evidence of the connection between stress fibers and perimeter. Notably, when stress fibers are present, they span the entire cell-substrate interface, which has an area far greater than that of the cell-cell interface. It is therefore reasonable to infer that the forces of cell-substrate traction are far larger than the forces in the cortex at cell-cell adhesions. This explains how tractions affect the average cell perimeter even though they act inside the cell's boundaries rather than at its periphery.

In all our experiments, the data suggest that tractions produced by stress fibers are the primary driver behind cell 

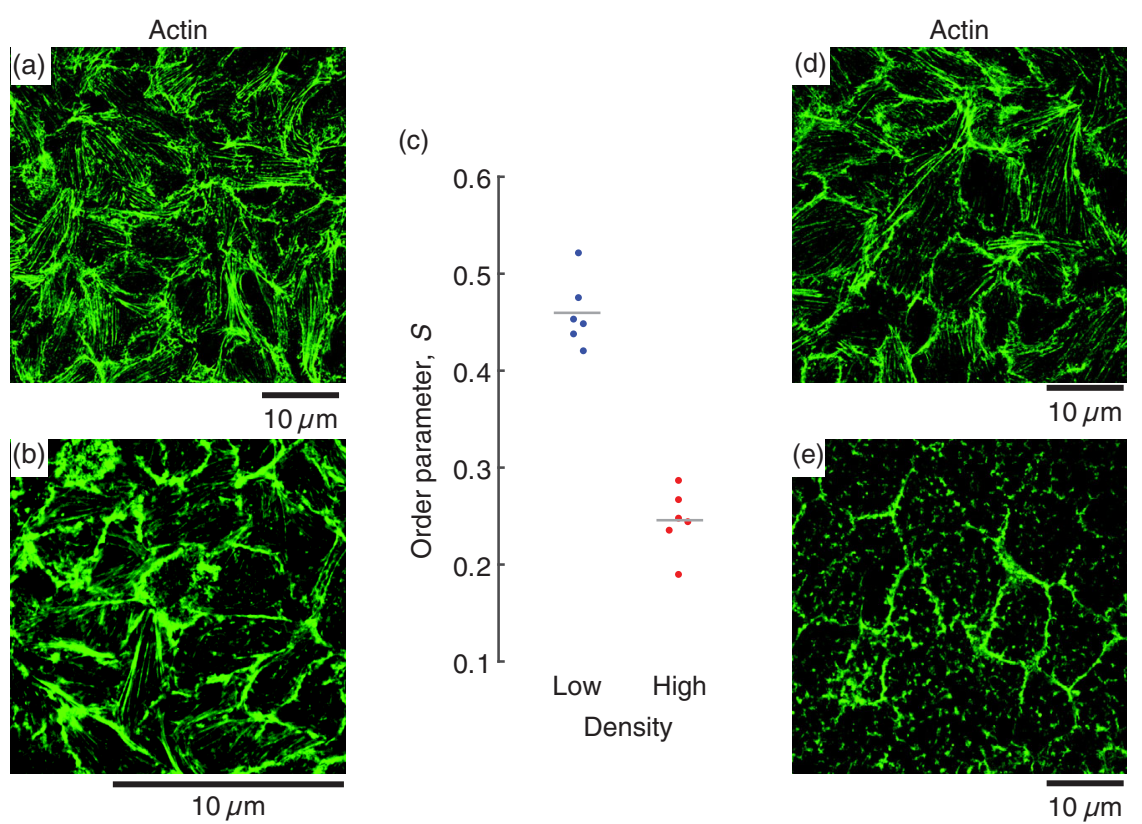

(f)

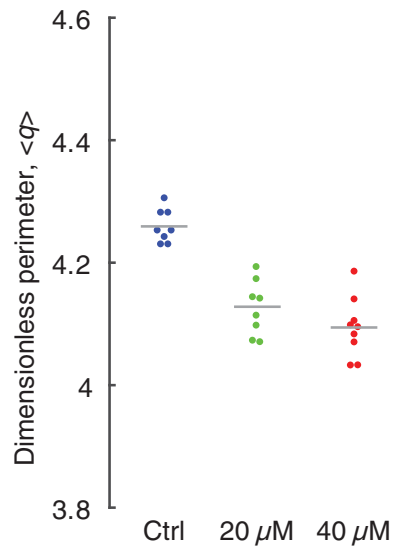

FIG. 5. Effect of density on stress fiber organization. (a),(b) Confocal images of actin stress fibers in low- $\left(1200 \mathrm{cells} / \mathrm{mm}^{2}\right)$ (a) and high-density $\left(4200\right.$ cells $/ \mathrm{mm}^{2}$ ) (b) monolayers. (c) Order parameter $S$ quantifying average stress fiber alignment in different monolayers. $S$ is lower at high density compared to low density $(p<0.001)$. (d),(e) Confocal images of actin stress fibers of control cells (d) and cells treated with $40 \mu \mathrm{M}$ of SMIFH2 (e) (the density for both panels is approximately $1200 \mathrm{cells} / \mathrm{mm}^{2}$ ). (f) The average dimensionless perimeter $\langle q\rangle$ decreases with SMIFH2 treatment $(p<0.001)$ for both treatments compared to the control.

perimeter and rearrangements. We therefore hypothesize that the effect of density on the cell perimeter [Fig. 1(h)] and rearrangements [Figs. 1(e)-1(g)] could be reversed by increasing cell-substrate tractions. To test this hypothesis, we seed islands at two different densities, 1200 cells $/ \mathrm{mm}^{2}$ and 2600 cells $/ \mathrm{mm}^{2}$. As in Fig. 1, cells at higher density have a lower perimeter $\langle q\rangle$ and rearrange less than cells at low density [Figs. 6(a)-6(d)]. To increase cell-substrate tractions in the higher-density islands, we treat them with the Rho activator CN03 $(2 \mu \mathrm{g} / \mathrm{mL})$, which increases stress fiber alignment [Figs. 15(a)-15(d)] and increases both the rms traction and the traction imbalance by a factor of approximately 3 [Figs. 6(e), 6(f), 15(e), and 15(f)]. The treatment also increases the perimeter $\langle q\rangle$ such that it becomes statistically indistinguishable from the perimeter of cells at low density [Fig. 6(a)]. As Figs. 3 and 4 suggest that traction affects cell perimeter, we expect the same to be true here. An alternative explanation is that the $\mathrm{CNO}$ treatment affects cell line tension by decreasing cortical tension, increasing cell-cell adhesions, or both. To rule out this explanation, we quantify fluorescence of PMLC and E-cadherin at cell peripheries and measure recoil velocity after laser ablation. There is no significant change in fluorescent intensities or recoil velocities (Fig. 16), implying no change in line tension. Another alternative explanation is that the $\mathrm{CNO} 03$ treatment directly increases the cell perimeters, causing cell elongation, which is known to stimulate stress fiber activity and increase tractions $[43,44]$.
To rule out this explanation, we examine phase contrast images of cells at various time points after the $\mathrm{CNO3}$ treatment. The cell perimeters appear to increase around 180-230 min after the treatment, whereas the traction increases earlier, around $120 \mathrm{~min}$ after the treatment (Fig. 17). Data from multiple experiments show that the rms traction and traction imbalance become statistically larger 120 and $180 \mathrm{~min}$ after the treatment, whereas the perimeter becomes statistically larger than the control $230 \mathrm{~min}$ after the treatment [Figs. 6(e)-6(g)]. Therefore, the CNO3 increases the traction before the perimeter. The finding that $\mathrm{CNO} 3$ increases traction before the perimeter gives additional evidence supporting the notion that tractions and the associated stress fibers that produce them are the underlying factor affecting the perimeter.

The Rho activator also significantly increases cell rearrangements in the high-density cell islands, as quantified by the exponents of MSD and CRMSD [Figs. 6(c), 6(d), and 18]. The difference is most notable in the exponent of CRMSD $\alpha_{\mathrm{CR}}$, which increases to a value above that of the low-density islands [Fig. 6(d)]. This observation can be explained by the fact that average cell displacement is lower at high density compared to low density [Fig. 6(b)], which tends to increase the exponent of CRMSD for the highdensity islands compared to low-density islands. Together, the data demonstrate that by activating stress fibers to increase cell-substrate tractions, the effect of cell density on the perimeter and rearrangements can be reversed. 

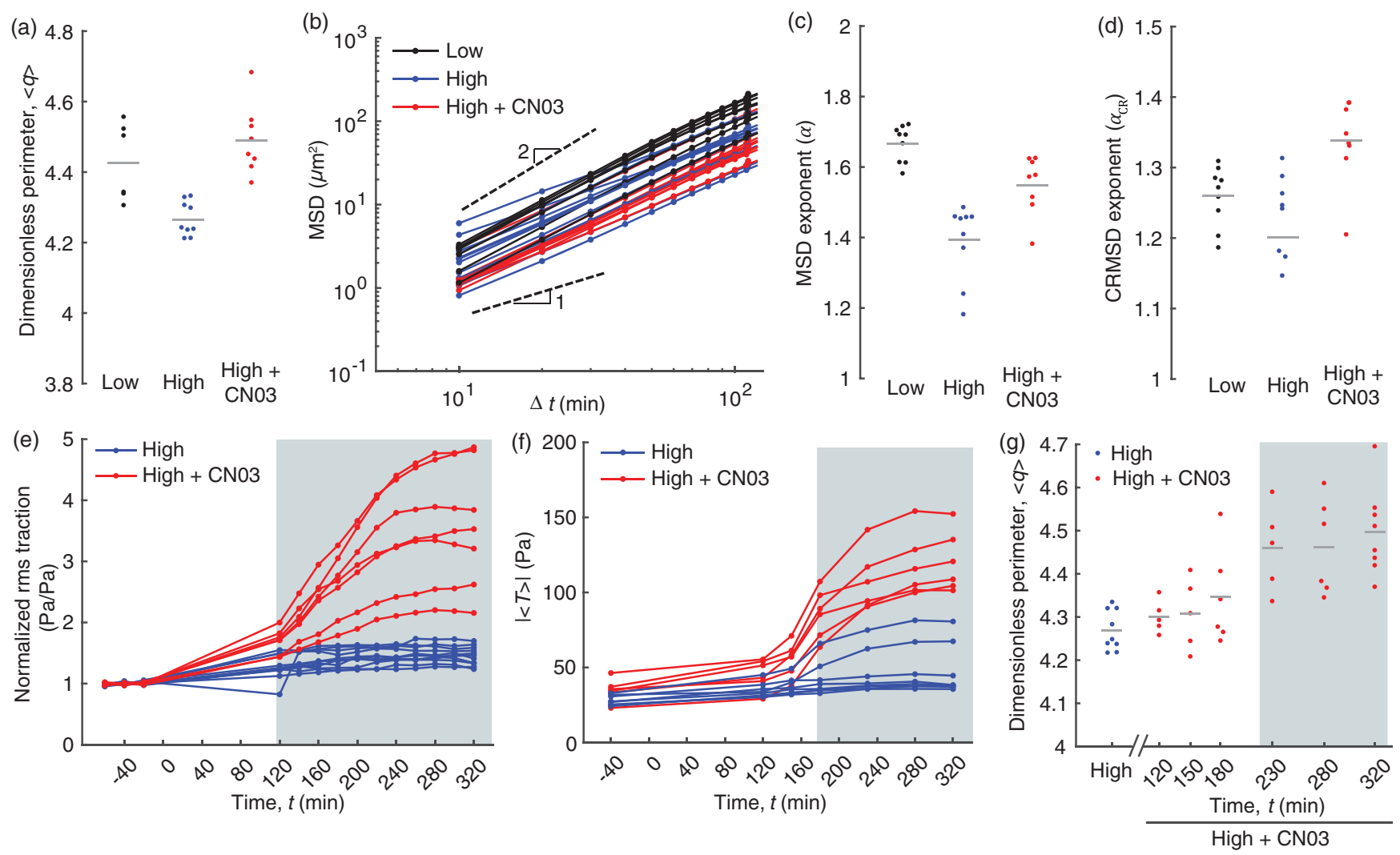

FIG. 6. Increasing cell-substrate tractions reverses the effect of density on the cell perimeter and cell rearrangements. (a) Average perimeter $\langle q\rangle$ for cells in islands of low density $\left(1200\right.$ cells $\left./ \mathrm{mm}^{2}\right)$, high density $\left(2600\right.$ cells $\left./ \mathrm{mm}^{2}\right)$, or high density with the Rho activator CN03 $(2 \mu \mathrm{g} / \mathrm{mL})$. The low-density and CN03 groups are statistically different from the high-density groups $(p<0.005)$. (b) MSD for low-density, high-density, and CN03-treated high-density islands. (c) Exponent $\alpha$ of the MSD for low-density and CN03treated high-density islands is different from the untreated high-density islands $(p<0.005)$. (d) Exponent $\alpha$ of CRMSD for low-density and CN03-treated high-density islands is different from the untreated high-density islands $(p<0.01)$. (e) Average rms traction for highdensity and CN03-treated high-density islands measured over time before $(t<0)$ and after $(t>0)$ treatment with vehicle control or CN03. Shading shows time points for which tractions produced by the CN03-treated cells are statistically different from the high-density control $(p<0.001)$. (f) Average traction imbalance for high-density and CN03-treated high-density islands measured over time before $(t<0)$ and after $(t>0)$ treatment with vehicle control or CN03 for a circular region of radius $R=12 \mu \mathrm{m}$. Shading shows time points for which $\mathrm{CN} 03$-treated cells are statistically different from the high-density control $(p<0.001)$. (g) Average cell perimeter becomes statistically larger than the control at $230 \mathrm{~min}$ after the CN03 treatment $(p<0.005)$.

\section{DISCUSSION}

Though the relationship between force and migration in a cell monolayer is still poorly understood, a useful framework is the analogy between cell monolayers and glassy materials, specifically the transition between fluidlike and solidlike states [45]. Perhaps the strongest evidence for this analogy is the observation that cells appear to transition toward solidlike states with increasing density $[4,5,7,11-14]$. Here, we hypothesize that changing density affects the motion by altering either the line tension at the cell-cell interface or the traction produced by the stress fibers. We observe increasing density to reduce tractions, average cell perimeter $\langle q\rangle$, and cell rearrangements. Similarly, tractions, perimeters, and rearrangements are reduced by inhibitors of actomyosin contraction. In contrast to current understanding, the changes in the perimeter and rearrangements appear unrelated to the line tension associated with cortical tension and cell-cell adhesions. This leads to the hypothesis that traction is the primary driver of cell rearrangements. To test this hypothesis, we activate actomyosin contraction in cell islands of high density, which causes cell perimeters and rearrangements to match those in monolayers at low density, thereby reversing the apparent effect of density on cell rearrangements. We conclude that the perimeter and rearrangements in an adherent epithelial monolayer are controlled primarily by tractions produced by the stress fibers.

Our findings provide insights to interpret recent theories and experiments which showed that cell perimeter controls a transition between solidlike and fluidlike states in a cell monolayer $[6,15,18-26]$. These observations on the importance of perimeter focus attention on the force-supporting components at the periphery of each cell, namely, the cortex and the cell-cell adhesions, which together generate 
a line tension that acts like a surface tension [6,15,18-27]. Here we study the line tension at each cell periphery by recoil velocity after laser ablation, imaging phosphorylated myosin in the cortex, and imaging E-cadherin at the cell-cell adhesions. According to current understanding [6,15,18-27], both increased recoil velocity and increased phosphorylated myosin imply greater cortical tension, which reduce the perimeter; increased E-cadherin implies greater adhesion, which increases the perimeter. Phosphorylated myosin and E-cadherin are unchanged by increasing the cell density (Fig. 2), even as the perimeter is reduced [Fig. 1(g)]. In separate experiments, blebbistatin and cytochalasin D each reduce the cortical tension (Fig. 3), which is expected to increase the perimeter. By contrast, the perimeter is reduced by these treatments [Figs. 3(i)-3(j)]. Together, these observations suggest that either current understanding of the relationship between line tension and perimeter is incorrect or some factor other than line tension at the cell periphery affects the cell perimeters. Our study suggests that cell perimeters are affected by another factor: traction.

The data show a strong connection between cell-tosubstrate traction, aligned stress fibers, and cell perimeter. Though it is clear that stress fibers and traction are closely related, the underlying cause of the increased perimeter is unclear. It may be that increased cell perimeter results from the stress fibers, which may alter the internal forces within each cell, thereby polarizing that cell along a specific direction. Alternatively, the increased perimeter may result from the fact that imbalanced traction alters the balance of forces at each cell-cell interface, as studied by a theoretical model [21]. In this case, the relationship between perimeter and traction is an emergent phenomenon, resulting from the balance of forces between cells rather than within each cell. This distinction is perhaps not so important, however, because either case points to the same result: Greater tractions and greater forces in the stress fibers produce greater cell rearrangements within the cell layer.

The notion that stress fibers and traction are the key drivers of cell shapes and rearrangements in a monolayer provides insight into a previous observation in collective cell migration on the relationship between contraction and motion $[6,28]$. Greater contraction would presumably cause greater cortical tension, which would reduce both perimeter and rearrangements. By contrast, experiments observed greater contraction to be associated with larger perimeter and more cell rearrangements $[6,28]$. This observation was hypothesized to result from a change in the adhesion molecules between cells [6]. Our data suggest a different resolution - that the greater perimeter and cell rearrangements occur not because of the forces at the cell-cell interfaces but rather because of the stress fibers and cellsubstrate tractions. Indeed, greater cell rearrangement was observed previously to coincide with greater traction $[6,28]$, which is consistent with a theoretical model that considered traction in addition to cortical tension and adhesion [21]. Our findings also provide insight into a recent study that proposed density to affect rearrangements by modulating cell-cell adhesions, which were suggested to create an effective friction that increased with density [7]. Though it is possible that friction could affect the migration, our finding that cell perimeters are governed primarily by stress fibers and traction rather than cortical tension or adhesion suggests that tractions produced by stress fibers are the primary drivers of cell rearrangements.

A logical extension of the observation that cell-substrate tractions control the perimeter and rearrangement more so than cortical tension or cell-cell adhesion is that the energy associated with traction is greater than that associated with cortical tension or adhesion. Cells with large traction and large perimeter have well-established, highly ordered stress fibers, which are present across the entire area of each cell (e.g., Fig. 5). By contrast, contraction in the cortex is present only at the periphery of each cell. As the area associated with the stress fibers is much larger than that associated with cortical tension, the magnitude of force produced in the stress fibers is likely to be far greater than in the cortex, causing the stress fibers, and therefore, the tractions that they apply to the substrate, to be the dominant factor affecting cell perimeter and rearrangement. We expect this reasoning to apply to any epithelial cell type that applies large tractions to the substrate. Consistent with this, monolayers of human bronchial epithelial and MCF10A cells appear to show relationships between tractions, perimeter, and collective migration [6,28]. Additionally, stress fiber activity is related to elongation of ovarian follicle cells in Drosophila development [46]. If there are no stress fibers, the cortical tension and cell-cell adhesions could control the cell perimeters and rearrangements. This may be the case in those aspects of development where there is little traction between cell and substrate [18,47-50]. Cell spheroids also have no cell-substrate tractions, and, consistent with our reasoning, cell perimeters are controlled by adhesions and cortical tension in cell spheroids [51].

Recent studies have proposed a jamming phase diagram with different axes representing the factors controlling whether or not cells rearrange positions with their neighbors $[14,21,45,52]$. Density has been proposed as one axis $[14,45,52]$, but our data show that increasing the tractions can reverse the apparent effect of density on cell perimeter and rearrangements. This finding can be explained by a theoretical model that proposed an additional axis of the phase diagram proportional to traction [21]. Other theoretical models have suggested that the axes of cell density and contraction are coupled but nevertheless separate $[14,52]$. Although we do not rule out the possibility that cell density is a distinct axis on the phase diagram, the experiments here demonstrate that effects of cell density on cell shape and rearrangements can be explained largely by cell traction produced by stress fibers. Another proposed axis of the phase diagram is related to the line tension $\Gamma$ resulting from the balance of adhesion and cortical tension 
$[6,21]$. Though we similarly do not rule out the existence of this axis, our results demonstrate that the cell perimeters and rearrangements are far more sensitive to traction. We expect that in systems where tractions are small, such as an embryo, the situation may be reversed, with adhesion and cortical tension playing a dominant role. It is likely that still other axes of the phase diagram await to be discovered. The importance of stress fibers and traction observed here provides a starting point for future experiments that answer these questions.

\section{MATERIALS AND METHODS}

\section{A. Cell culture}

Madin-Darby canine kidney type II cells expressing either green fluorescent protein (GFP) with a nuclear localization signal or Lifeact-GFP are supplied by the laboratory of Professor David Weitz, Harvard University. The cells are maintained in low-glucose Dulbecco's modified Eagle's medium (Cellgro 10-014-CV, Corning Inc., Corning, NY) with $10 \%$ fetal bovine serum (Corning) and $1 \%$ G418 (Corning) in an incubator at $37^{\circ} \mathrm{C}$ and $5 \% \mathrm{CO}_{2}$. Cells with nuclear GFP are used for traction, migration, and immunofluorescence experiments, whereas Lifeact cells are used for laser ablation experiments. For experiments using blebbistatin, cytochalasin $\mathrm{D}$, and $\mathrm{SMIFH}$, cell medium is replaced with medium containing $2 \%$ fetal bovine serum 3-4 h before the treatments. For experiments with $\mathrm{CNO3}$, cell medium is replaced with medium containing $1 \%$ fetal bovine serum one day before the treatment. Time-lapse experiments are performed for $4-6 \mathrm{~h}$ to maintain a relatively constant density.

\section{B. Polyacrylamide substrates}

Polyacrylamide gels with Young's modulus of $6 \mathrm{kPa}$ and thickness of $150 \mu \mathrm{m}$ are prepared with fluorescent particles located at the top. A gel solution of $5.5 \%$ weight/volume $(w / v)$ acrylamide (Biorad Laboratories, Hercules, CA) and $0.2 \% \mathrm{w} / v$ bisacrylamide (Biorad) is prepared, and $20 \mu \mathrm{L}$ is pipetted onto no. 1.5 glass-bottom dishes (Cellvis, Mountain View, CA). A glass coverslip (18-mm-diameter circle) is placed on top of each gel and removed after the gel solution is polymerized. Then, a second gel solution with composition of the first gel plus $0.036 \% \mathrm{w} / \mathrm{v}$ fluorescent particles (diameter $0.5 \mu \mathrm{m}$, carboxylate modified; Life Technologies) is prepared, and $20 \mu \mathrm{L}$ is pipetted on the polymerized first gel. Again, a coverslip is placed on top, and the dishes are centrifuged upside down to localize the fluorescent particles to the top of the second gel. The top surface of the second gel is functionalized with type I rat tail collagen (BD Biosciences, Franklin Lakes, NJ; $0.01 \mathrm{mg} / \mathrm{mL}, 1-2 \mathrm{~mL}$ per 18-mm-diameter gel) using the covalent cross-linker sulfo-SANPAH (Pierce Biotechnology, Waltham, MA).

\section{Micropatterning confined cellular islands}

Polydimethysiloxane (PDMS) (Sylgard 184, Dow Corning, Midland, MI) is poured onto plastic dishes to cure for $4 \mathrm{~h}$ on a hot plate at $70^{\circ} \mathrm{C}$ to make (400-600)- $\mu \mathrm{m}$ thick sheets. These sheets are cut into $16-\mathrm{mm}$ circular masks, and biopsy punches (1 mm diameter) are used to make holes in the PDMS masks. The masks are sterilized with $70 \%$ ethanol and incubated overnight at room temperature in 2\% Pluronic F-127 (Sigma-Aldrich, St. Louis, MO) to prevent cell adhesion to the masks. The masks are placed on the polyacrylamide gels before functionalizing with sulfo-SANPAH and collagen, thereby constraining the collagen to circular patterns on the gels. Next, $300 \mu \mathrm{L}$ of cell solution of concentration $0.5 \times 10^{6}$ cells $/ \mathrm{mL}$ is pipetted onto the masks and incubated at $37^{\circ} \mathrm{C}$ for $2 \mathrm{~h}$. The masks are then removed, and the patterned cell islands are placed in the incubator at $37^{\circ} \mathrm{C}$ and $5 \% \mathrm{CO}_{2}$ until they attain the desired density for imaging.

\section{Widefield microscopy}

Images of the cell islands and the fluorescent particles are captured every 5 or 10 min using phase contrast and fluorescent modes of an Eclipse Ti microscope (Nikon, Melville, NY) with a $10 \times$ numerical aperture 0.5 objective and an Orca Flash 4.0 digital camera (Hamamatsu, Bridgewater, NJ) running NIS-Elements Ar software (Nikon). The imaging environment is maintained at $37^{\circ} \mathrm{C}$ and $5 \% \mathrm{CO}_{2}$ using an $\mathrm{H} 301$ stage-top incubator with a UNO controller (Okolab USA Inc., San Bruno, CA). After the time-lapse experiments, the cells are removed from the polyacrylamide substrates by incubating in $0.05 \%$ trypsin for $20 \mathrm{~min}$, and images of the fluorescent particles are collected; these images provide a traction-free reference state for computing cell-substrate tractions.

\section{E. Traction force microscopy}

The term "traction" in this manuscript refers to the vector field of in-plane force per area applied by the cells to the substrate. To quantify tractions, cell-induced displacements of the fluorescent particles are measured using fast iterative digital image correlation (FIDIC) [53] using $32 \times 32$ pixel subsets centered on a grid with a spacing of 8 pixels $(5 \mu \mathrm{m})$. Tractions are subsequently computed using unconstrained Fourier-transform traction microscopy [36] accounting for the finite substrate thickness $[37,38]$. The computed traction has components in the $x$ and $y$ directions $T_{x}$ and $T_{y}$, respectively. The rms of the traction is computed according to the expression $\left[\left\langle T_{x}^{2}+T_{y}^{2}\right\rangle\right]^{1 / 2}$, where the angle brackets \langle\rangle indicate a mean over all positions. The average traction imbalance is computed for each grid point according to the expression $\left|\sum_{i}\left(\vec{T}_{i} A\right) / \sum_{i} A\right|$, where $\vec{T}_{i}$ is the traction vector, $A$ is the (constant) area over which each traction vector acts, and the sum is a vector sum over all locations $i$ within a circle of radius $R$ from the grid point of interest. 
The computation is repeated for $R$ ranging from 9 to $90 \mu \mathrm{m}$. This is repeated for every grid point at least $60 \mu \mathrm{m}$ from the edge of each cell island and averaged over the cell island. The symbol $|\langle T\rangle|$ is used to represent the average traction imbalance.

\section{F. Cell velocities and trajectories}

Cell velocities are measured using FIDIC [53] from phase contrast images of cell islands. Consecutive images are correlated, and the resulting displacements are divided by time to compute velocity. Subsets of $48 \times 48$ pixels are used with a spacing of 12 pixels $(8 \mu \mathrm{m})$. The cell island boundaries are detected using the MATLAB 2015a code written based on Ref. [54].

The cell displacements computed with FIDIC are used to calculate cell trajectories as described in Refs. [6,28]. The calculation begins with an initial evenly spaced grid, with the distance between grid points matching the average cell size. Displacements from FIDIC are interpolated to each grid point, and the interpolated values are added to the coordinates of each grid point to give an estimate of each cell's position at the next time point. This process is repeated for each time point of the experiment, thereby creating a list of points for each cell corresponding to that cell's trajectory.

\section{G. Mean-square displacements}

As a metric for cell rearrangement, the MSD is computed according to the equation

$\operatorname{MSD}(\Delta t)=\left\langle\left|u_{i}(t, \Delta t)\right|^{2}\right\rangle=\left\langle\left|r_{i}(t+\Delta t)-r_{i}(t)\right|^{2}\right\rangle$,

where $r_{i}(t)$ is the position of the $i$ th cell at time $t, u_{i}(t, \Delta t)$ is the displacement of the $i$ th cell for time interval $\Delta t$, and the brackets \langle\rangle denote an average over all cells and all possible starting time points $t$. In some Figs. 7, 12, and 18, the mean-square displacement is shown for individual cells, in which case, the average is taken over all $t$; for these figures, the notation $\mathrm{MSD}_{i}$ is used to indicate that the curves represent individual cells. As tractions are nearly constant for a time span of $120 \mathrm{~min}$, the MSD is computed over a time span of $120 \mathrm{~min}$.

\section{H. Cage-relative mean-square displacements}

As a second metric for rearrangements, the CRMSD is computed as explained in Ref. [29]. The CRMSD quantifies each cell's motion relative to the local motion of its $N$ nearest neighbors according to the equation

$$
\operatorname{CRMSD}(\Delta t)=\left\langle\left|u_{i}(t, \Delta t)-u_{i}^{\text {cage }}(t, \Delta t)\right|^{2}\right\rangle,
$$

where $u_{i}^{\text {cage }}(t, \Delta t)$ is the average displacement of the nearest $N$ neighbors to cell $i$, and $N$ is equal to 4,10 , or
20, as described in the text. The CRMSD is computed over the same time span as the MSD.

\section{Chemical treatments}

Chemical treatments are blebbistatin (Sigma-Aldrich), cytochalasin D (Sigma-Aldrich), and CN03 (Cytoskeleton, Inc., Denver, CO). Stock solutions of blebbistatin, cytochalasin D, and CN03 are prepared at $20 \mathrm{mM}, 2 \mathrm{mM}$, and $0.1 \mathrm{~g} / \mathrm{L}$, respectively, all dissolved in DMSO except CNO3 in water. The stock solutions are diluted in phosphatebuffered saline (PBS) to obtain the desired concentrations for the experiments.

\section{J. Immunofluorescence and confocal microscopy}

For immunofluorescent labeling, MDCK cells are seeded to form monolayers or islands of the required confluence and treated with the chemical of interest. After $3 \mathrm{~h}$, the cells are rinsed twice with PBS and fixed with 4\% paraformaldehyde in PBS for 20 min. The cells are washed with Tris-buffered saline twice for 5 min each. For actin staining, the cells are incubated in $0.1 \%$ Triton $\mathrm{X}-100$ for $5 \mathrm{~min}$ at room temperature and then placed in 3-5 units/mL solution of Phalloidin Dylight 594 (Life Technologies catalog no. 21836). For E-cadherin and myosin staining, the cells are treated with E-cadherin rabbit antibody (1:200 ratio; Cell Signaling, Danvers, MA, catalog no. 24E10) or phospho-Myosin Light Chain 2 (Ser 19) rabbit antibody (1:100 ratio; Cell Signaling catalog no. 3671). After washing the cells, the Alexa 647 antirabbit secondary antibody (1:400 ratio; Life Technologies, catalog no. A-21245) is used for fluorescent staining.

Fluorescently labeled cells are imaged with a Nikon $A 1 R+$ confocal microscope with a $40 \times$ NA 1.15 waterimmersion objective with a step size of $0.5 \mu \mathrm{m}$ using NISElements Ar software (Nikon). For imaging cortical actin, E-cadherin, and PMLC, image stacks are captured near the apex of the cells, and a maximum intensity projection having a thickness of $3 \mu \mathrm{m}$ is computed. For imaging stress fibers, image stacks are captured at the base of the cells, and a maximum intensity projection having thickness of $1.5 \mu \mathrm{m}$ is computed. Representative images shown in the figures are cropped and pseudocolored using ImageJ.

\section{K. Measurement of dimensionless cell perimeter}

A maximum intensity projection of the confocal images of F-actin near the apex of the cell layer gives images of the cell boundaries. The cells are then segmented using SEEDWATER SEGMENTER [55], and the coordinates of each cell boundary are identified using the BWBOUNDARIES function in MATLAB 2015a. Vertices defined as locations where three or more cells meet are identified from the coordinates of the cell boundaries. Each cell perimeter is computed by summing the distances between the vertices. 
Each cell area is computed from the identified vertices using the POLYAREA function in MATLAB 2015a. The dimensionless cell perimeter $q$ is then computed according to $q=P A^{-1 / 2}$. Finally, the mean of $q,\langle q\rangle$, is computed over $100-150$ cells.

\section{E-cadherin and PMLC analysis}

Fluorescent intensities of E-cadherin and PMLC are quantified in the cell cortex located at the cell peripheries. To account for background signal, the median of each image is subtracted from that image. Peripheral locations are defined by dilating the segmented images used to measure the cell perimeter by $1 \mu \mathrm{m}$; pixels within the dilated images are defined as being at the cell peripheries and used to quantify intensities of E-cadherin and PMLC. For comparisons between control and treatments, the intensities are normalized by the average of the control.

\section{Order parameter}

Stress fibers are quantified within the bulk of each cell. To determine the positions for analysis, the same segmentation as used for E-cadherin and PMLC is used, except stress fibers are defined to be outside the dilated cell boundaries. We quantify actin stress fiber alignment in each cell using an order parameter $S$. For this, we calculate the angle $\theta$ at each pixel inside a cell of interest using the OrientationJ plugin [56] in ImageJ and compute the average of the angles $\theta_{m}$. The order parameter $S$ for the cell is defined as

$$
S=2\left[\left\langle\cos ^{2}\left(\theta-\theta_{m}\right)\right\rangle-1 / 2\right],
$$

where the brackets \langle\rangle indicate an average over all positions within the cell. The order parameter $S$ attains a value of 1 for fully aligned stress fibers and 0 for random alignment.

\section{N. Laser ablation}

Laser ablation is performed on a CSU-X spinning disk confocal microscope (Yokogawa) mounted on an Eclipse Ti base with a $60 \times$ NA 1.4 oil immersion objective (Nikon) and imaged with a Zyla sCMOS camera (Andor), all run by the IQ3 acquisition software (Andor). MDCK Lifeact cells are seeded to confluence on glass substrates as in Ref. [31], and cell-cell edges are visualized with the 488-nm laser line. A Micropoint laser at $405 \mathrm{~nm}$ is used to ablate cell-cell edges using a single 0.06 -s pulse at $65 \%$ laser power. An image is captured before ablation to determine the initial distance $d(0)$, and images are captured every $5 \mathrm{~s}$ from 1 to $41 \mathrm{~s}$ after the ablation. The distance $d(t)$ between the vertices of each ablated cell edge is measured manually for each time point. The initial recoil velocity is defined as the slope of the plot of $d(t)-d(0)$ vs time from 0 to $11 \mathrm{~s}$, which is computed using a least-mean-square fitting that is constrained to go through the origin.

\section{O. Statistical analysis}

Unless otherwise noted, each colored dot or line in the plots is an average taken over a different cell island or monolayer and represents an independent biological sample. Tractions, MSD, and CRMSD are measured in cell islands, whereas the cell perimeter, fluorescent intensities, and order parameter are computed in larger cell monolayers. Statistical comparisons between groups are performed using a two-sided Student's $t$-test or, for multiple groups, one-way analysis of variance with Tukey's correction for multiple comparisons. Analyses are performed in MATLAB 2015a.

\section{ACKNOWLEDGMENTS}

We thank B. Burkel and M. Graham for comments on the manuscript. Confocal microscopy is performed at the Biochemistry Optical Core and the Materials Science Center of the University of Wisconsin-Madison. This work is supported by National Science Foundation Grant No. CMMI-1660703. 


\section{APPENDIX}
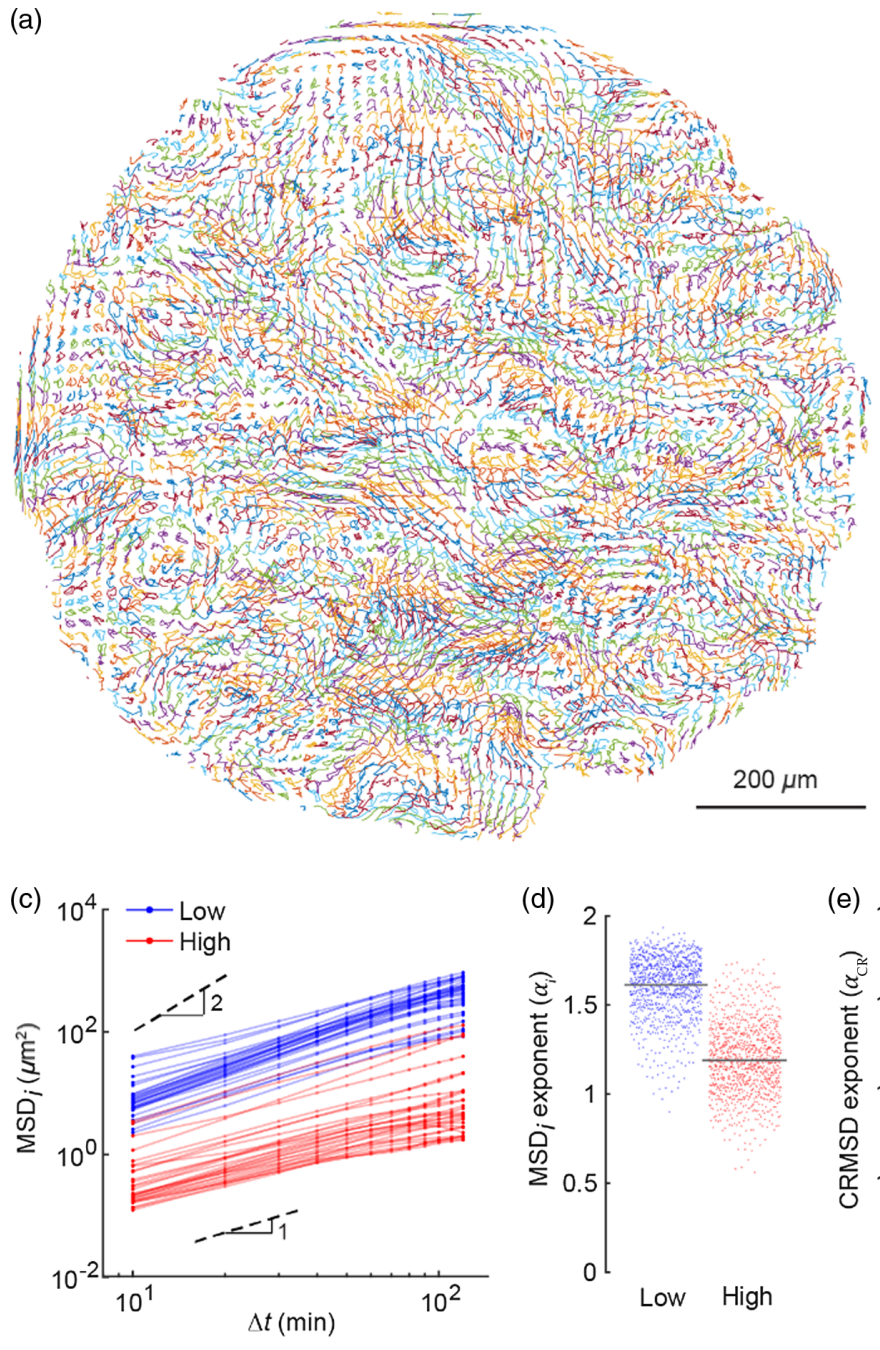
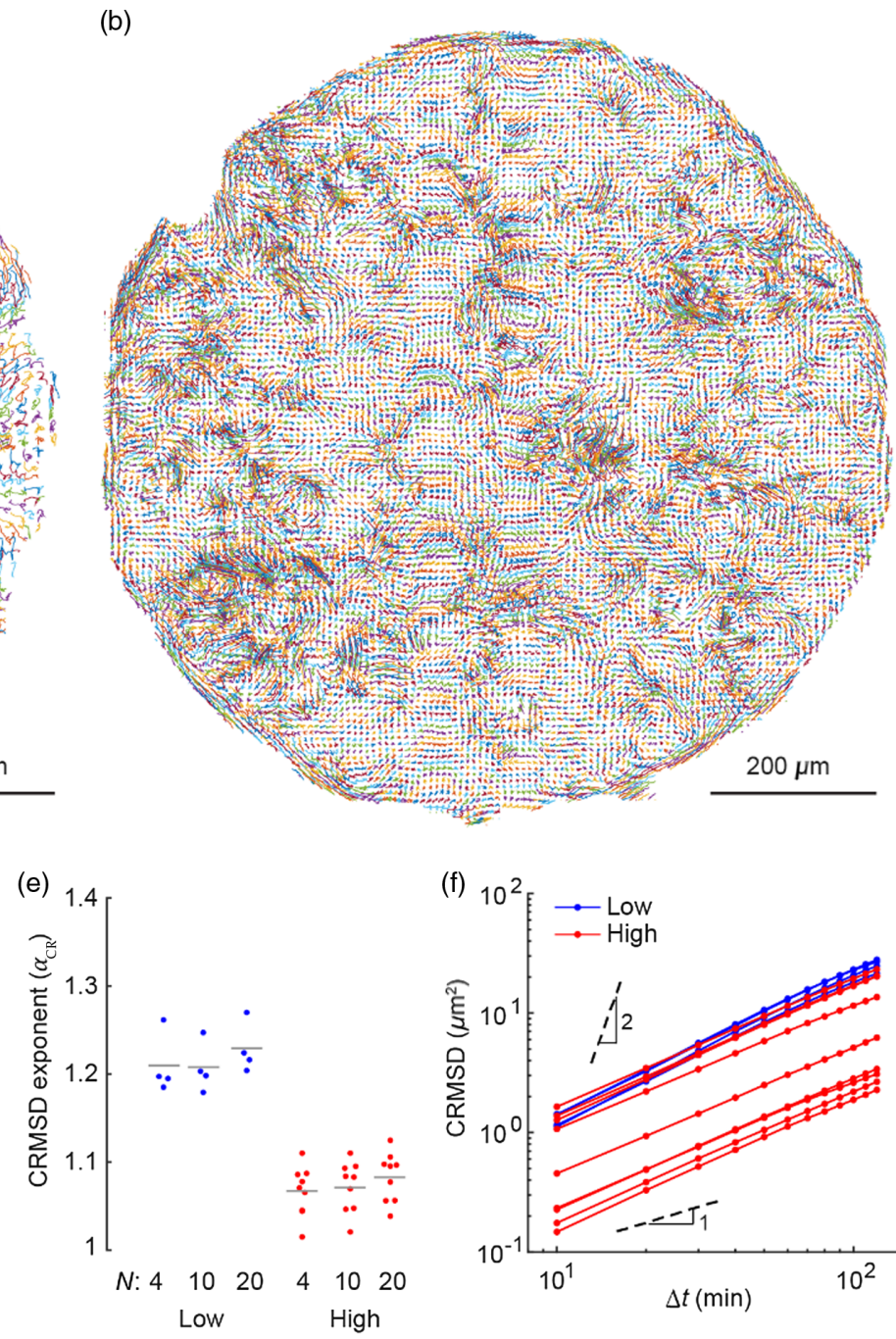

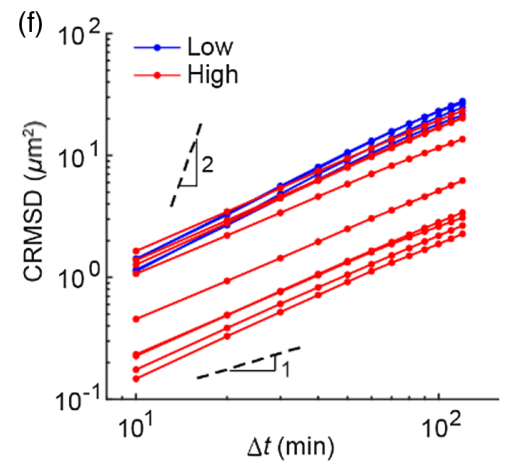

FIG. 7. Effect of density on collective motion. Cell trajectories computed over a time span of $8 \mathrm{~h}$ in low- $\left(1200 \mathrm{cells} / \mathrm{mm}^{2}\right)(\mathrm{a})$ and high-density (4200 cells $/ \mathrm{mm}^{2}$ ) (b) islands. These are the same cell islands as in Figs. 1(a) and 1(c). (c) Mean-square displacement computed from trajectories of selected cells $\left(\mathrm{MSD}_{i}\right)$ from single islands of low and high density. (d) Exponent $\alpha_{i}$ of the $\mathrm{MSD}_{i}$ curves in panel (c) $(p<0.001)$. Each dot in panel (d) represents an individual cell. (e) Exponent $\alpha_{\mathrm{CR}}$ of the CRMSD curves for different cell pack sizes $N$ for cell islands of low and high densities. (f) CRMSD of low- and high-density islands for $N=20$. 

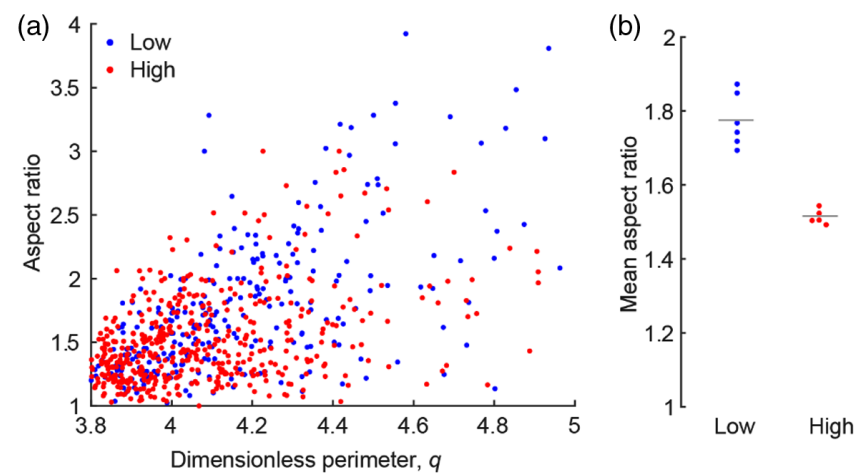

FIG. 8. Correlation between the aspect ratio and dimensionless perimeter. (a) Scatter plot between the aspect ratio and dimensionless perimeter for cells at low (1200 cells $\left./ \mathrm{mm}^{2}\right)$ and high density $\left(4200\right.$ cells $\left./ \mathrm{mm}^{2}\right)$. Each dot corresponds to a different cell. Aspect ratio is computed from the cell vertices using the REGIONPROPS command of MATLAB 2015a. (b) Mean aspect ratio is smaller at high density compared to low density $(p<0.001)$. Each dot corresponds to an average of 100-150 cells in a different field of view.

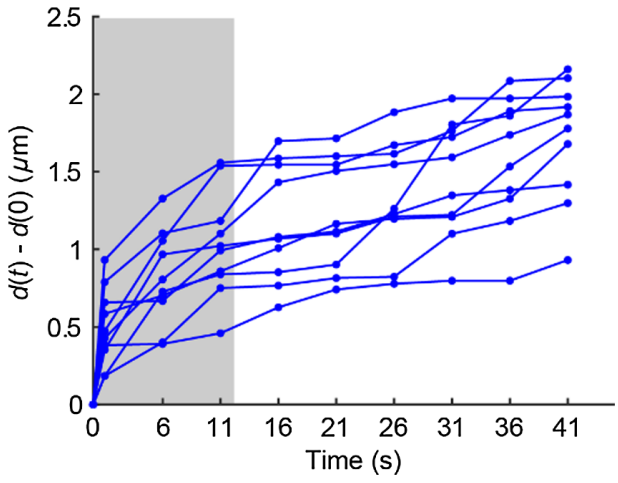

FIG. 9. Recoil in vertices that connect a cell edge after laser ablation. Change in distance $d(t)-d(0)$ between vertices after laser ablation of cells in a monolayer of low density $\left(1200\right.$ cells $\left./ \mathrm{mm}^{2}\right)$. Initial recoil velocity is computed by fitting a line to data points in the gray region corresponding to $t=0-11 \mathrm{~s}$. Each line corresponds to an ablated cell edge.
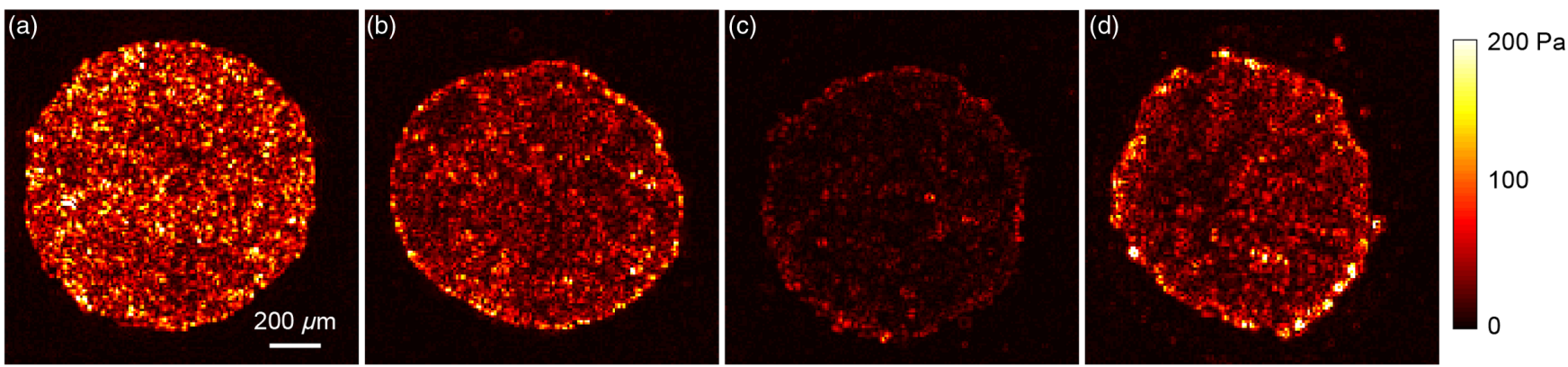

FIG. 10. (a),(b) Color maps of traction magnitude in low-density (1200 cells $\left./ \mathrm{mm}^{2}\right)$ (a), high-density (4200 cells $\left./ \mathrm{mm}^{2}\right)(\mathrm{b})$, blebbistatin-treated (1200 cells $\left./ \mathrm{mm}^{2}\right)(\mathrm{c})$, and cytochalasin D-treated $\left(1200\right.$ cells $\left./ \mathrm{mm}^{2}\right)(\mathrm{d})$ islands. 


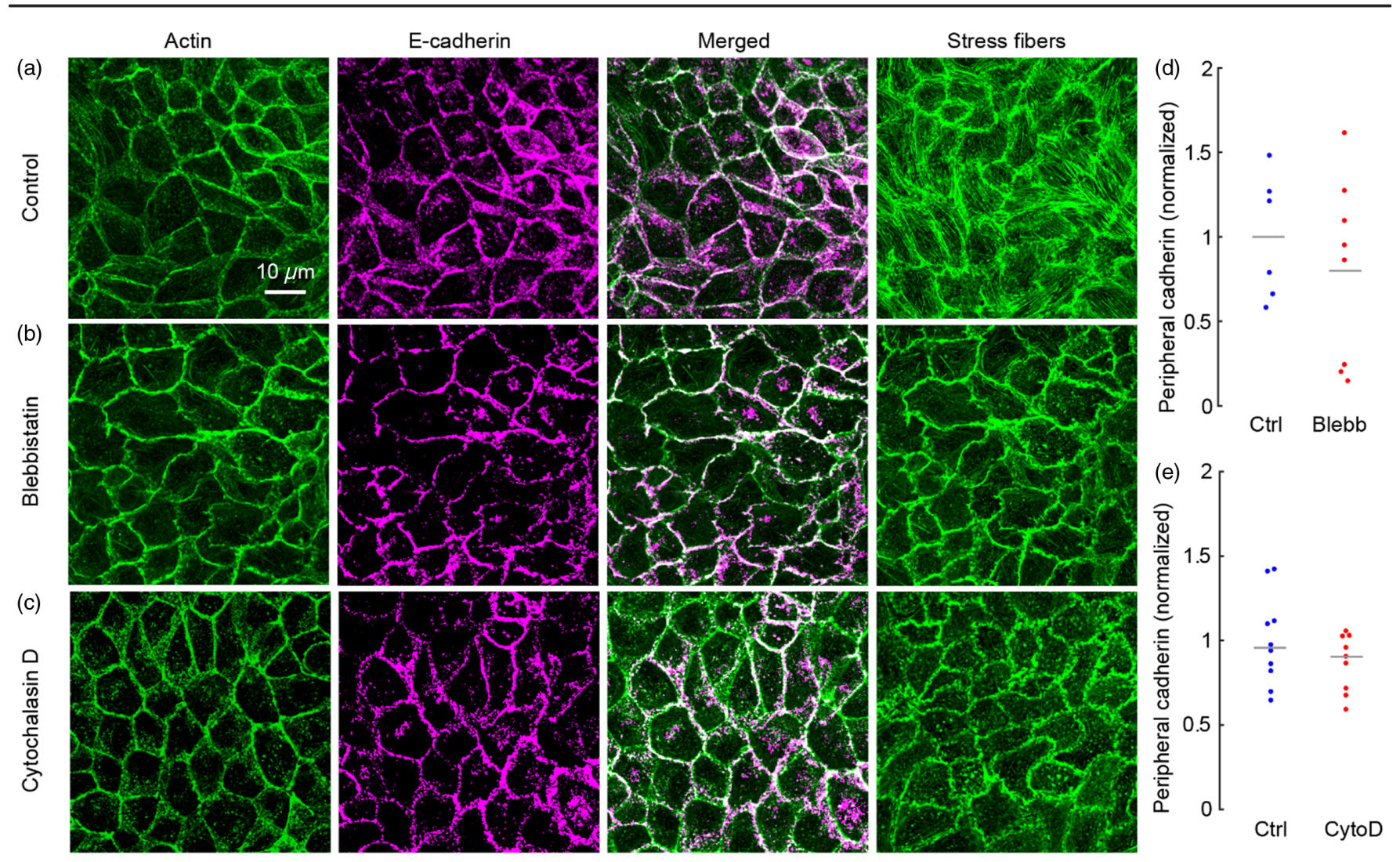

FIG. 11. Effect of blebbistatin and cytochalasin D on E-cadherin and stress fibers. (a)-(c) Confocal images of cortical actin, E-cadherin, and actin stress fibers for the control cell monolayers (a) and cell monolayers treated with $20 \mu \mathrm{M}$ of blebbistatin (b) or $0.05 \mu \mathrm{M}$ of cytochalasin D (c). (d),(e) Peripheral E-cadherin intensities are unaffected by $20 \mu \mathrm{M}$ of blebbistatin [(d) $p=0.45]$ or $0.05 \mu \mathrm{M}$ of cytochalasin $\mathrm{D}$ [(e) $p=0.23$. The cell density in all panels is 1200 cells $/ \mathrm{mm}^{2}$. 

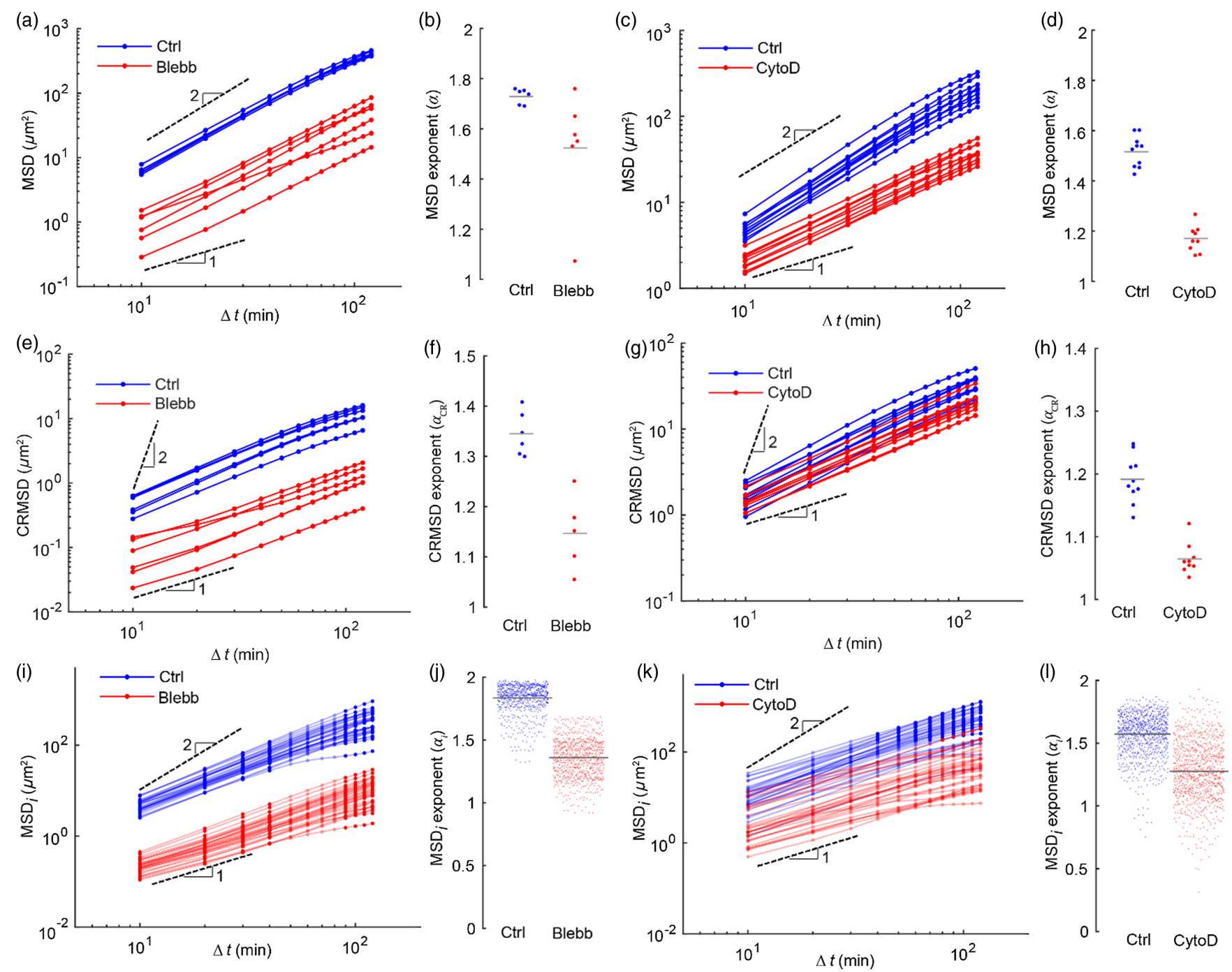

FIG. 12. Cell rearrangements decrease with inhibition of actomyosin contractility. (a),(c) MSD of control cell islands and islands treated with $20 \mu \mathrm{M}$ of blebbistatin (a) or $0.05 \mu \mathrm{M}$ of cytochalasin D (c). (b),(d) Exponent $\alpha$ of MSD decreases after treating with $20 \mu \mathrm{M}$ of blebbistatin [(b) $p<0.05$ ] or $0.05 \mu \mathrm{M}$ of cytochalasin D [(d) $p<0.001]$. (e),(g) CRMSD of control cell islands and islands treated with $20 \mu \mathrm{M}$ of blebbistatin (e) or $0.05 \mu \mathrm{M}$ of cytochalasin D (g). (f),(h) Exponent $\alpha_{\mathrm{CR}}$ of CRMSD decreases after treating with $20 \mu \mathrm{M}$ of blebbistatin [(f) $p<0.001$ ] or $0.05 \mu \mathrm{M}$ of cytochalasin D [(h) $p<0.001]$. (i), (k) $\mathrm{MSD}_{i}$ for selected cell trajectories in the control islands and islands treated with $20 \mu \mathrm{M}$ of blebbistatin (i) or $0.05 \mu \mathrm{M}$ of cytochalasin D (k). (j),(1) Exponent $\alpha_{i}$ of $\mathrm{MSD}_{i}$ of cell trajectories in the control islands and islands treated with $20 \mu \mathrm{M}$ of blebbistatin [(j) $\left.p<0.001\right]$ or control islands and islands treated with $0.05 \mu \mathrm{M}$ of cytochalasin $\mathrm{D}$ [(1) $p<0.001]$. Dots in panels (j) and (l) represent individual cells. The cell density in all panels is 1200 cells $/ \mathrm{mm}^{2}$. 


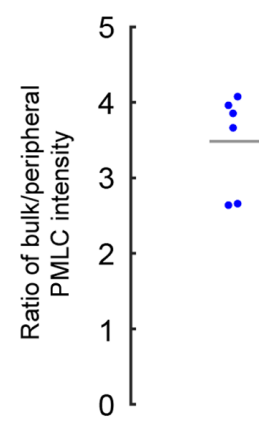

FIG. 13. Ratio of total PMLC intensity in the bulk and peripheral region. Peripheral PMLC is quantified as described in Sec. IV. Bulk PMLC is quantified in the same region as the stress fibers, namely, at the base of the cell layer and away from the cell peripheries. The cell density is 1200 cells $/ \mathrm{mm}^{2}$.
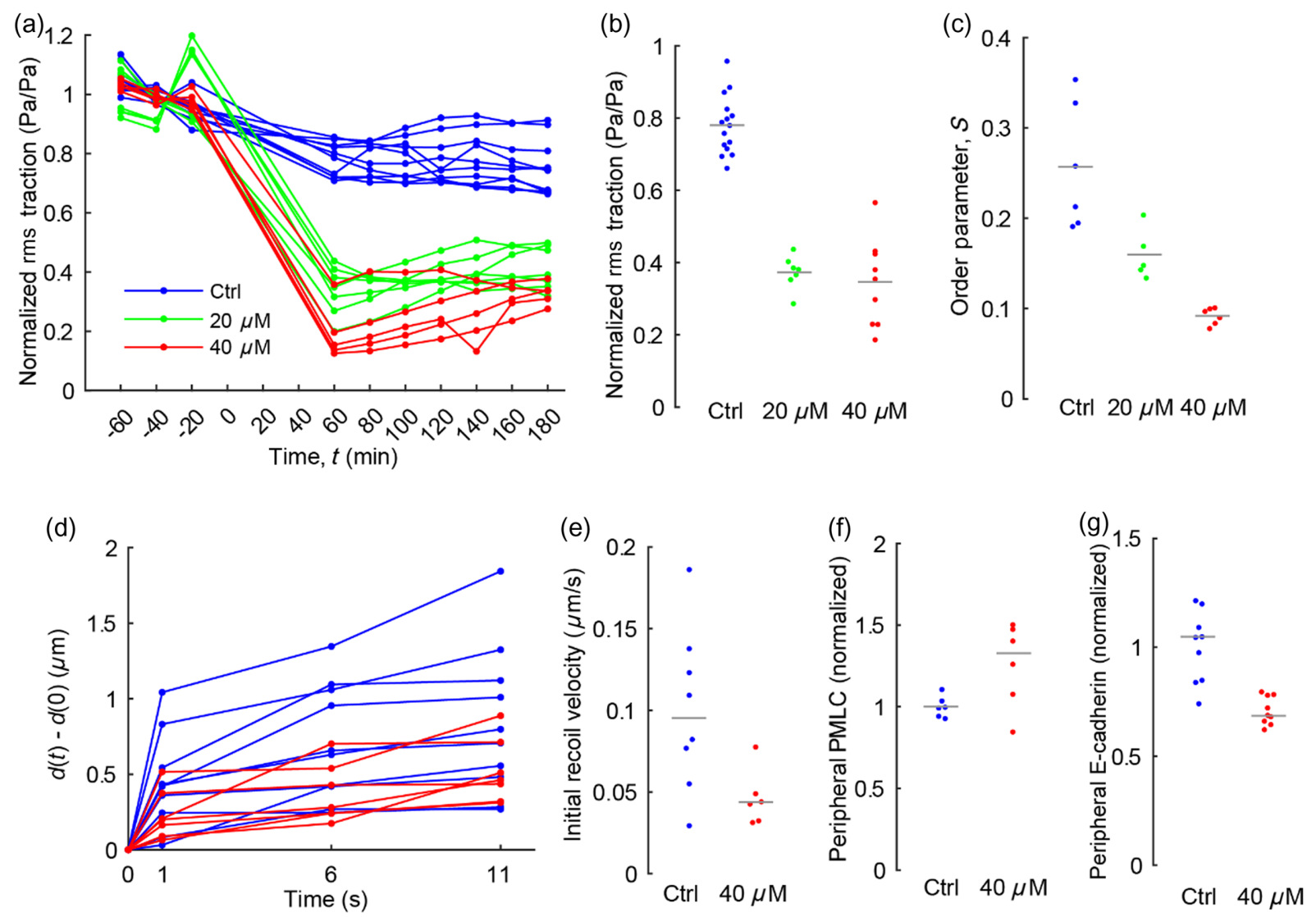

FIG. 14. Stress fiber inhibition. (a) Average rms traction for each island is measured over time before $(t<0)$ and after $(t>0)$ treating with the formin inhibitor SMIFH2. For each island, the rms traction is normalized by its average value before the treatment. (b) Normalized rms traction for $t=60-180 \mathrm{~min}$. Relative to control, both concentrations reduce the rms traction $(p<0.001)$. (c) Order parameter $S$ decreases after stress fiber inhibition $(p<0.001)$. The cell density is 1200 cells $/ \mathrm{mm}^{2}$. (d) Change in distance between the vertices of an ablated edge $d(t)-d(0)$ for the control and cells treated with $40 \mu \mathrm{M}$ of SMIFH2. (e) Initial recoil velocity after laser ablation is decreased by SMIFH2 $(p<0.01)$. (f) Peripheral PMLC fluorescent intensity is increased by SMIFH2 $(p<0.05)$. (g) Peripheral E-cadherin intensity is decreased by SMIFH2 $(p<0.01)$. 
(a)

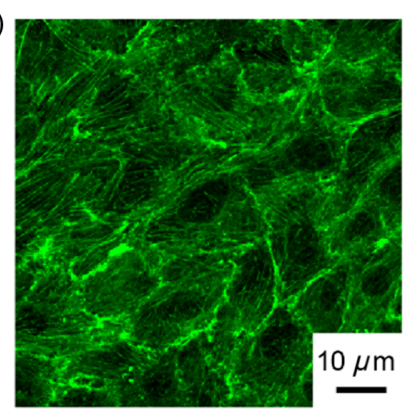

(b)

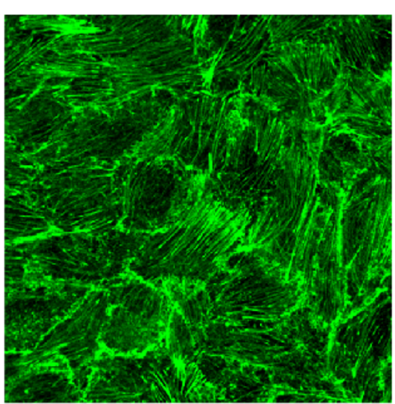

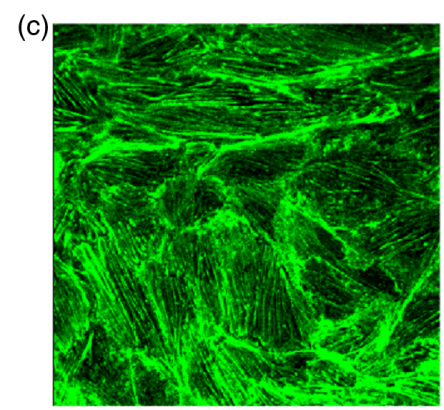

(1)

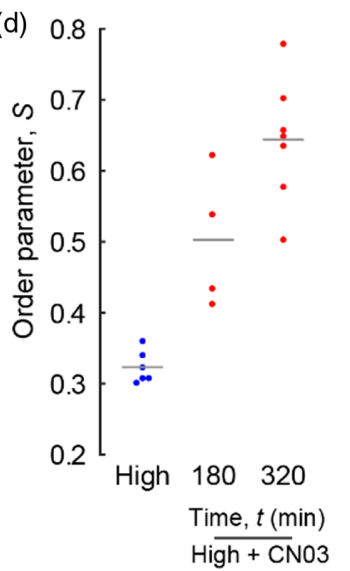

(e)
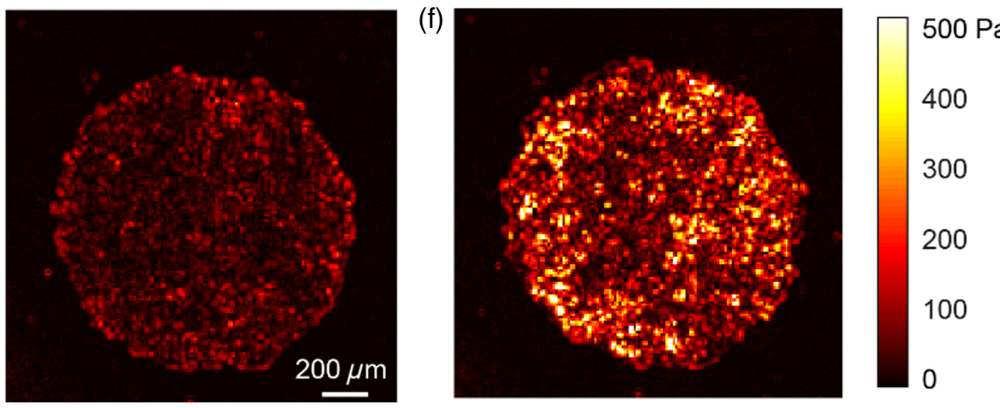

FIG. 15. Rho activator CN03 increases stress fibers and tractions. (a)-(c) Confocal images of actin stress fibers for the control cells at high density (a) and cells at high density at $t=180 \mathrm{~min}$ (b) or $320 \mathrm{~min}$ (c) after treating with CN03 (2 $\mu \mathrm{g} / \mathrm{mL}$ ). (d) Order parameter for high density is smaller than for high density treated with CN03 ( $p<0.001$ for both groups compared to the high-density control). (e),(f) Color maps of traction magnitude of high density (e) and high density treated with CN03 at $t=320$ min (f). Cell density for all data in this figure is 2600 cells $/ \mathrm{mm}^{2}$.

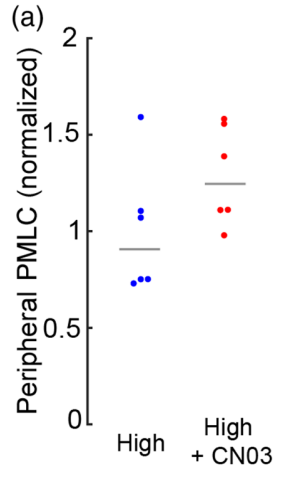

(b)

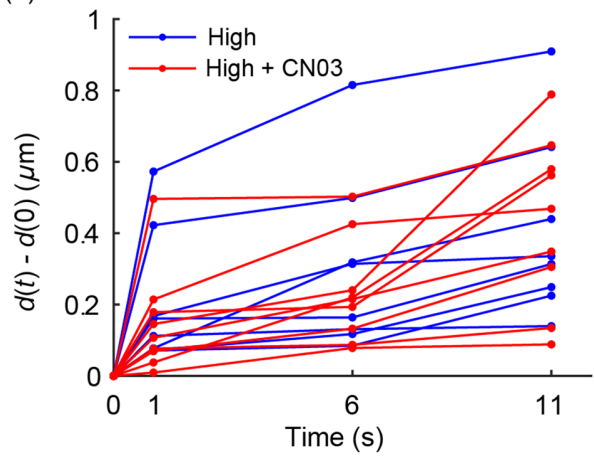

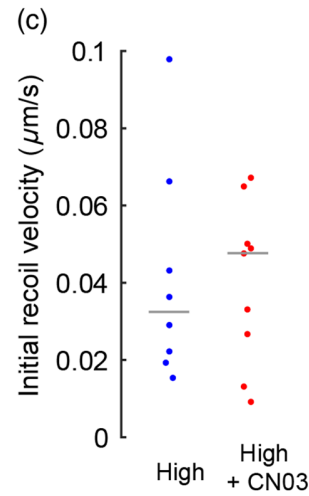

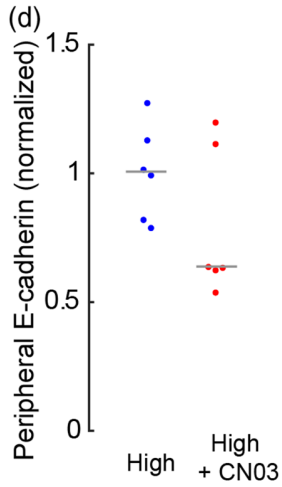

FIG. 16. Effect of CN03 $(2 \mu \mathrm{g} / \mathrm{mL})$ on line tension. (a) Fluorescent intensity of peripheral PMLC $(p=0.12)$. (b),(c) Change in distance $d(t)-d(0)$ after laser ablation (b) and initial recoil velocity [ $p=0.86$ (c)]. (d) Fluorescent intensity of peripheral E-cadherin $(p=0.15)$. Cell density for all data in this figure is 2600 cells $/ \mathrm{mm}^{2}$. 

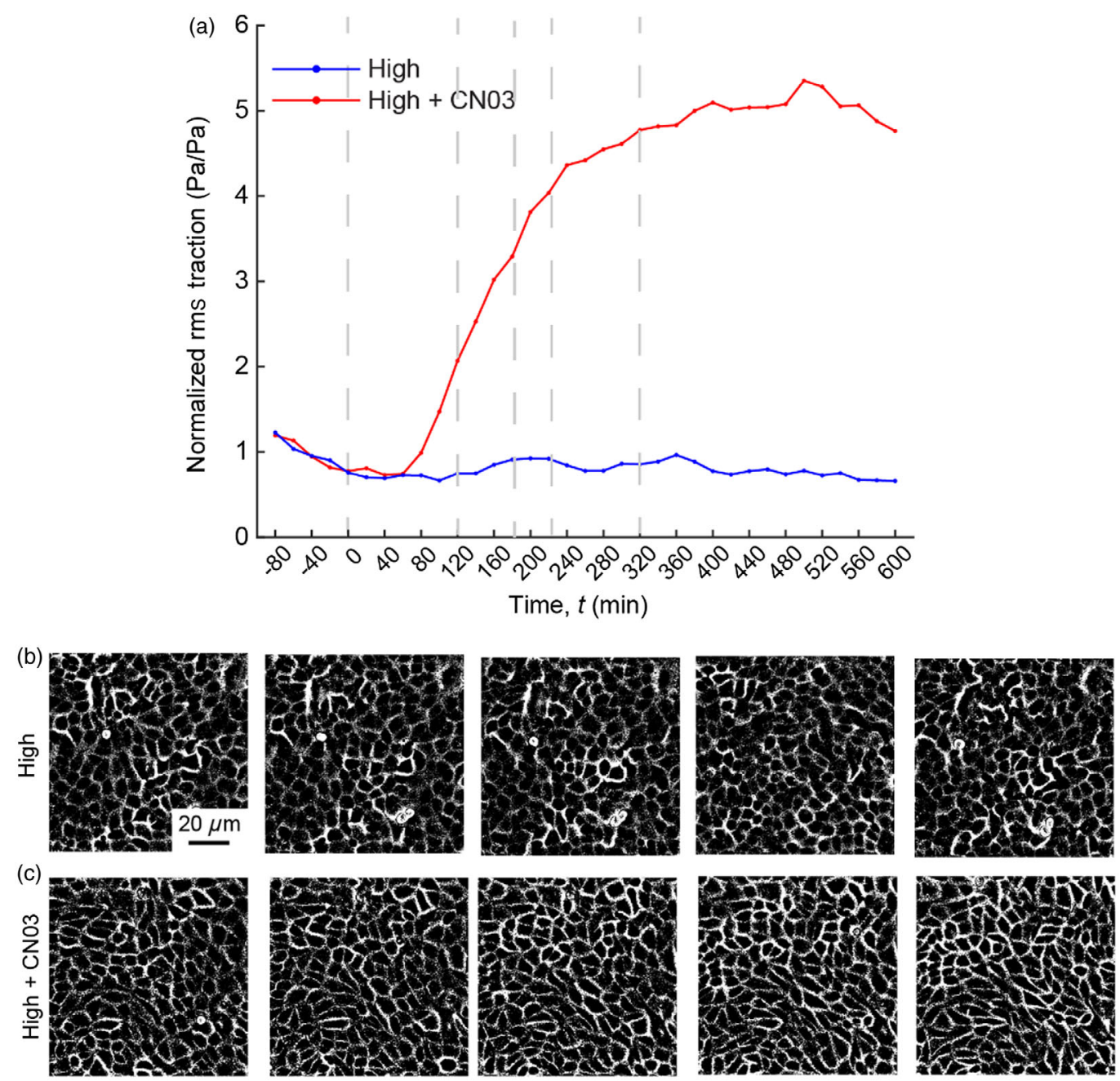

$t=0 \mathrm{~min}$

$t=120 \mathrm{~min}$

$t=180 \mathrm{~min}$

$t=230 \mathrm{~min}$

$t=320 \mathrm{~min}$

FIG. 17. Changes in traction precede changes in dimensionless cell perimeter. (a) Average rms traction for the high-density island $\left(2600\right.$ cells $\left./ \mathrm{mm}^{2}\right)$ and high-density islands treated with CN03 $(2 \mu \mathrm{g} / \mathrm{mL})$ measured over time before $(t<0)$ and after $(t>0)$ treatment with vehicle control or CNO3. (b),(c) Phase contrast images of control cells (b) and CN03-treated cells (c) at different times during the treatment.
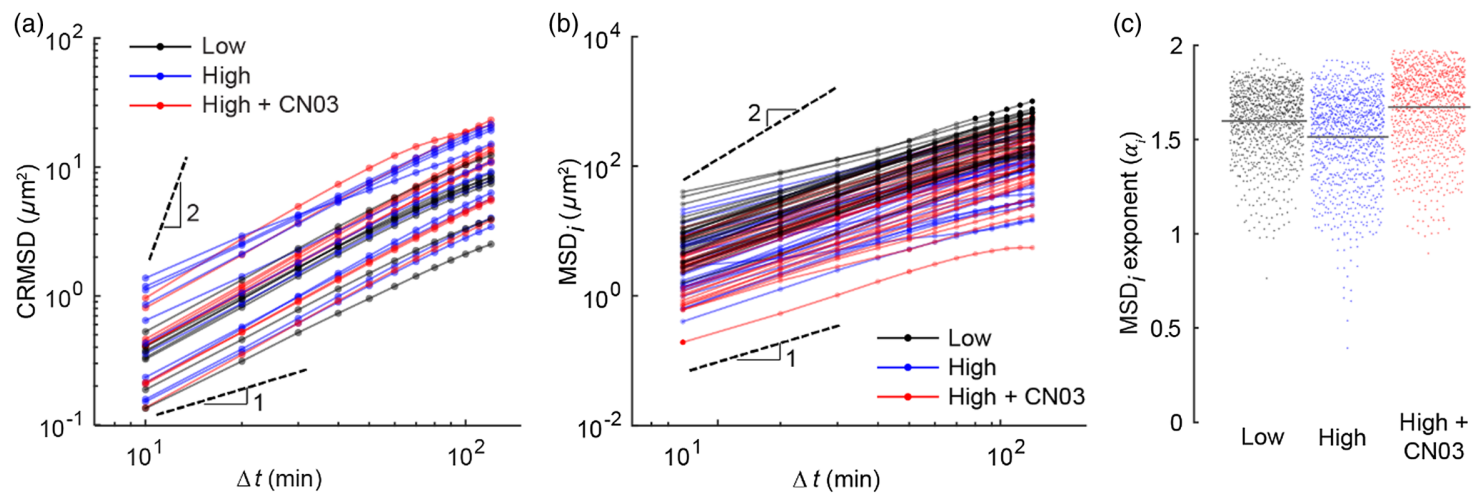

FIG. 18. Rho activator CN03 increases cell rearrangements. (a) CRMSD for islands of low density, high density, and high density treated with CNO3 $(2 \mu \mathrm{g} / \mathrm{mL})$. (b) $\mathrm{MSD}_{i}$ for selected cell trajectories in islands of low density $\left(1200\right.$ cells $\left./ \mathrm{mm}^{2}\right)$, high density (2600 cells $\left./ \mathrm{mm}^{2}\right)$, and high density treated with CN03 islands $(2 \mu \mathrm{g} / \mathrm{mL})$. (c) Exponent $\alpha_{i}$ of $\mathrm{MSD}_{i}$ of cell trajectories in islands of low density, high density, and high density treated with CN03 $(2 \mu \mathrm{g} / \mathrm{mL})$. Each dot represents an individual cell. 
[1] P. Friedl and D. Gilmour, Collective Cell Migration in Morphogenesis, Regeneration and Cancer, Nat. Rev. Mol. Cell Biol. 10, 445 (2009).

[2] L. Oswald, S. Grosser, D. M. Smith, and J. A. Käs, Jamming Transitions in Cancer, J. Phys. D 50, 483001 (2017).

[3] A. Mongera, P. Rowghanian, H. J. Gustafson, E. Shelton, D. A. Kealhofer, E. K. Carn, F. Serwane, A. A. Lucio, J. Giammona, and O. Campàs, A Fluid-to-Solid Jamming Transition Underlies Vertebrate Body Axis Elongation, Nature (London) 561, 401 (2018).

[4] T. E. Angelini, E. Hannezo, X. Trepat, M. Marquez, J. J. Fredberg, and D. A. Weitz, Glass-like Dynamics of Collective Cell Migration, Proc. Natl. Acad. Sci. U.S.A. 108, 4714 (2011).

[5] K. D. Nnetu, M. Knorr, S. Pawlizak, T. Fuhs, and J. A. Käs, Slow and Anomalous Dynamics of an MCF-10A Epithelial Cell Monolayer, Soft Matter 9, 9335 (2013).

[6] J.-A. Park et al., Unjamming and Cell Shape in the Asthmatic Airway Epithelium, Nat. Mater. 14, 1040 (2015).

[7] S. Garcia, E. Hannezo, J. Elgeti, J.-F. Joanny, P. Silberzan, and N. S. Gov, Physics of Active Jamming during Collective Cellular Motion in a Monolayer, Proc. Natl. Acad. Sci. U.S.A. 112, 15314 (2015).

[8] L. Atia et al., Geometric Constraints during Epithelial Jamming, Nat. Phys. 14, 613 (2018).

[9] A. J. Liu and S. R. Nagel, Jamming Is Not Just Cool Any More: Nonlinear Dynamics, Nature (London) 396, 21 (1998).

[10] V. Trappe, V. Prasad, L. Cipelletti, P. N. Segre, and D. A. Weitz, Jamming Phase Diagram for Attractive Particles, Nature (London) 411, 772 (2001).

[11] D. T. Tambe et al., Collective Cell Guidance by Cooperative Intercellular Forces, Nat. Mater. 10, 469 (2011).

[12] A. Puliafito, L. Hufnagel, P. Neveu, S. Streichan, A. Sigal, D. K. Fygenson, and B. I. Shraiman, Collective and Single Cell Behavior in Epithelial Contact Inhibition, Proc. Natl. Acad. Sci. U.S.A. 109, 739 (2012).

[13] K. Doxzen, S. R. K. Vedula, M. C. Leong, H. Hirata, N. S. Gov, A. J. Kabla, B. Ladoux, and C. T. Lim, Guidance of Collective Cell Migration by Substrate Geometry, Integr. Biol. 5, 1026 (2013).

[14] A. J. Loza, S. Koride, G. V. Schimizzi, B. Li, S. X. Sun, and G. D. Longmore, Cell Density and Actomyosin Contractility Control the Organization of Migrating Collectives within an Epithelium, Mol. Biol. Cell 27, 3459 (2016).

[15] D. Bi, J. H. Lopez, J. M. Schwarz, and M. L. Manning, A Density-Independent Rigidity Transition in Biological Tissues, Nat. Phys. 11, 1074 (2015).

[16] R. Vincent, E. Bazellières, C. Pérez-González, M. Uroz, X. Serra-Picamal, and X. Trepat, Active Tensile Modulus of an Epithelial Monolayer, Phys. Rev. Lett. 115, 248103 (2015).

[17] J. Notbohm, S. Banerjee, K. J. C. Utuje, B. Gweon, H. Jang, Y. Park, J. Shin, J. P. Butler, J. J. Fredberg, and M. C. Marchetti, Cellular Contraction and Polarization Drive Collective Cellular Motion, Biophys. J. 110, 2729 (2016).

[18] R. Farhadifar, J.-C. Röper, B. Aigouy, S. Eaton, and F. Jülicher, The Influence of Cell Mechanics, Cell-Cell Interactions, and Proliferation on Epithelial Packing, Curr. Biol. 17, 2095 (2007).
[19] D. B. Staple, R. Farhadifar, J.-C. Röper, B. Aigouy, S. Eaton, and F. Jülicher, Mechanics and Remodelling of Cell Packings in Epithelia, Eur. Phys. J. E 33, 117 (2010).

[20] A. R. Noppe, A. P. Roberts, Z. Neufeld, A. S. Yap, and G. A. Gomez, Modelling Wound Closure in an Epithelial Cell Sheet Using the Cellular Potts Model, Integr. Biol. 7, 1253 (2015).

[21] D. Bi, X. Yang, M. C. Marchetti, and M. L. Manning, Motility-Driven Glass and Jamming Transitions in Biological Tissues, Phys. Rev. X 6, 021011 (2016).

[22] M. Chiang and D. Marenduzzo, Glass Transitions in the Cellular Potts Model, Europhys. Lett. 116, 28009 (2016).

[23] D. L. Barton, S. Henkes, C. J. Weijer, and R. Sknepnek, Active Vertex Model for Cell-Resolution Description of Epithelial Tissue Mechanics, PLoS Comput. Biol. 13, e1005569 (2017).

[24] X. Yang, D. Bi, M. Czajkowski, M. Merkel, M. L. Manning, and M. C. Marchetti, Correlating Cell Shape and Cellular Stress in Motile Confluent Tissues, Proc. Natl. Acad. Sci. U.S.A. 114, 12663 (2017).

[25] M. Moshe, M. J. Bowick, and M. C. Marchetti, Geometric Frustration and Solid-Solid Transitions in Model 2D Tissue, Phys. Rev. Lett. 120, 268105 (2018).

[26] M. Czajkowski, D. Bi, M. L. Manning, and M. C. Marchetti, Hydrodynamics of Shape-Driven Rigidity Transitions in Motile Tissues, Soft Matter 14, 5628 (2018).

[27] G. W. Brodland, The Differential Interfacial Tension Hypothesis (DITH): A Comprehensive Theory for the Self-Rearrangement of Embryonic Cells and Tissues, J. Biomech. Eng. 124, 188 (2002).

[28] C. Malinverno et al. Endocytic Reawakening of Motility in Jammed Epithelia, Nat. Mater. 16, 587 (2017).

[29] S.-Z. Lin, S. Ye, G.-K. Xu, B. Li, and X.-Q. Feng, Dynamic Migration Modes of Collective Cells, Biophys. J. 115, 1826 (2018).

[30] M. S. Hutson, Forces for Morphogenesis Investigated with Laser Microsurgery and Quantitative Modeling, Science 300, 145 (2003).

[31] S. Verma et al. A WAVE2-Arp2/3 Actin Nucleator Apparatus Supports Junctional Tension at the Epithelial Zonula Adherens, Mol. Biol. Cell 23, 4601 (2012).

[32] S. Curran, C. Strandkvist, J. Bathmann, M. de Gennes, A. Kabla, G. Salbreux, and B. Baum, Myosin II Controls Junction Fluctuations to Guide Epithelial Tissue Ordering, Dev. Cell 43, 480 (2017).

[33] Y. A. Miroshnikova et al., Adhesion Forces and Cortical Tension Couple Cell Proliferation and Differentiation to Drive Epidermal Stratification, Nat. Cell Biol. 20, 69 (2018).

[34] M. Krieg, Y. Arboleda-Estudillo, P.-H. Puech, J. Käfer, F. Graner, D. J. Müller, and C.-P. Heisenberg, Tensile Forces Govern Germ-Layer Organization in Zebrafish, Nat. Cell Biol. 10, 429 (2008).

[35] W. Engl, B. Arasi, L. L. Yap, J. P. Thiery, and V. Viasnoff, Actin Dynamics Modulate Mechanosensitive Immobilization of E-Cadherin at Adherens Junctions, Nat. Cell Biol. 16, 584 (2014).

[36] J. P. Butler, I. M. Tolić-Nørrelykke, B. Fabry, and J. J. Fredberg, Traction Fields, Moments, and Strain Energy That Cells Exert on Their Surroundings, Am. J. Physiol. Cell Physiol. 282, C595 (2002). 
[37] J. C. del Alamo, R. Meili, B. Alonso-Latorre, J. RodriguezRodriguez, A. Aliseda, R. A. Firtel, and J. C. Lasheras, Spatio-Temporal Analysis of Eukaryotic Cell Motility by Improved Force Cytometry, Proc. Natl. Acad. Sci. U.S.A. 104, 13343 (2007).

[38] X. Trepat, M. R. Wasserman, T. E. Angelini, E. Millet, D. A. Weitz, J. P. Butler, and J. J. Fredberg, Physical Forces during Collective Cell Migration, Nat. Phys. 5, 426 (2009).

[39] V. Maruthamuthu, B. Sabass, U. S. Schwarz, and M. L. Gardel, Cell-ECM Traction Force Modulates Endogenous Tension at Cell-Cell Contacts, Proc. Natl. Acad. Sci. U.S.A. 108, 4708 (2011).

[40] S. A. Rizvi, E. M. Neidt, J. Cui, Z. Feiger, C. T. Skau, M. L. Gardel, S. A. Kozmin, and D. R. Kovar, Identification and Characterization of a Small Molecule Inhibitor of ForminMediated Actin Assembly, Chem. Biol. 16, 1158 (2009).

[41] T. Isogai, R. van der Kammen, and M. Innocenti, SMIFH2 Has Effects on Formins and p53 That Perturb the Cell Cytoskeleton, Sci. Rep. 5, 9802 (2015).

[42] B. R. Acharya, S. K. Wu, Z. Z. Lieu, R. G. Parton, S. W. Grill, A. D. Bershadsky, G. A. Gomez, and A. S. Yap, Mammalian Diaphanous 1 Mediates a Pathway for E-Cadherin to Stabilize Epithelial Barriers through Junctional Contractility, Cell Rep. 18, 2854 (2017).

[43] M. Théry, A. Pépin, E. Dressaire, Y. Chen, and M. Bornens, Cell Distribution of Stress Fibres in Response to the Geometry of the Adhesive Environment, Cell Motil. Cytoskeleton 63, 341 (2006).

[44] P. W. Oakes, S. Banerjee, M. C. Marchetti, and M. L. Gardel, Geometry Regulates Traction Stresses in Adherent Cells, Biophys. J. 107, 825 (2014).

[45] M. Sadati, N. T. Qazvini, R. Krishnan, C. Y. Park, and J. J. Fredberg, Collective Migration and Cell Jamming, Differentiation (Berlin) 86, 121 (2013).

[46] L. He, X. Wang, H. L. Tang, and D. J. Montell, Tissue Elongation Requires Oscillating Contractions of a Basal Actomyosin Network, Nat. Cell Biol. 12, 1133 (2010).
[47] K. K. Chiou, L. Hufnagel, and B. I. Shraiman, Mechanical Stress Inference for Two Dimensional Cell Arrays, PLoS Comput. Biol. 8, e1002512 (2012).

[48] K. E. Kasza, D. L. Farrell, and J. A. Zallen, Spatiotemporal Control of Epithelial Remodeling by Regulated Myosin Phosphorylation, Proc. Natl. Acad. Sci. U.S.A. 111, 11732 (2014)

[49] G. W. Brodland, J. H. Veldhuis, S. Kim, M. Perrone, D. Mashburn, and M.S. Hutson, CellFIT: A Cellular ForceInference Toolkit Using Curvilinear Cell Boundaries, PLoS One 9, e99116 (2014).

[50] R. J. Tetley, M. F. Staddon, S. Banerjee, and Y. Mao, Tissue Fluidity Promotes Epithelial Wound Healing, Nat. Phys. 15, 1195 (2019).

[51] M. L. Manning, R. A. Foty, M. S. Steinberg, and E.-M. Schoetz, Coaction of Intercellular Adhesion and Cortical Tension Specifies Tissue Surface Tension, Proc. Natl. Acad. Sci. U.S.A. 107, 12517 (2010).

[52] S. Koride, A. J. Loza, and S. X. Sun, Epithelial Vertex Models with Active Biochemical Regulation of Contractility Can Explain Organized Collective Cell Motility, APL Bioeng. 2, 031906 (2018).

[53] E. Bar-Kochba, J. Toyjanova, E. Andrews, K.-S. Kim, and C. Franck, A Fast Iterative Digital Volume Correlation Algorithm for Large Deformations. Exp. Mech. 55, 261 (2015).

[54] K. K. Treloar and M. J. Simpson, Sensitivity of Edge Detection Methods for Quantifying Cell Migration Assays, PLoS One 8, e67389 (2013).

[55] D. N. Mashburn, H. E. Lynch, X. Ma, and M. S. Hutson, Enabling User-Guided Segmentation and Tracking of Surface-Labeled Cells in Time-Lapse Image Sets of Living Tissues, Cytometry 81A, 409 (2012).

[56] Z. Püspöki, M. Storath, D. Sage, and M. Unser, in Focus on Bio-Image Informatics, edited by W. H. De Vos, S. Munck, and J.-P. Timmermans (Springer International Publishing, New York, 2016), pp. 69-93. 